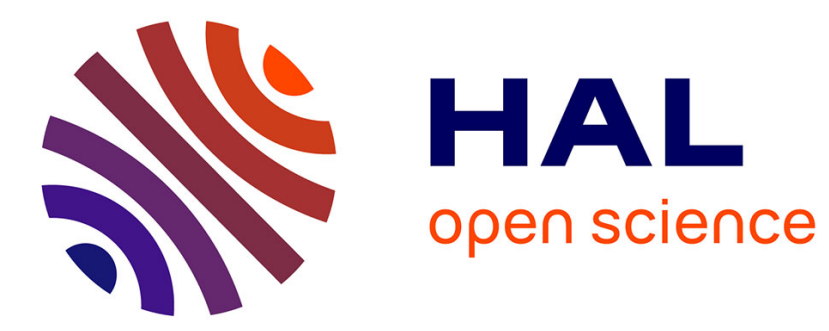

\title{
Ecologie territoriale et indicateurs pour un développement durable de la métropole parisienne
}

Johann Audrain, Mateo Cordier, Sylvie Faucheux, Marie-Françoise

Guyonnaud, Isabelle Nicolaï, Martin O’Connor

\section{- To cite this version:}

Johann Audrain, Mateo Cordier, Sylvie Faucheux, Marie-Françoise Guyonnaud, Isabelle Nicolaï, et al.. Ecologie territoriale et indicateurs pour un développement durable de la métropole parisienne. 2012. hal-00911712

\section{HAL Id: hal-00911712 \\ https://hal.science/hal-00911712}

Preprint submitted on 29 Nov 2013

HAL is a multi-disciplinary open access archive for the deposit and dissemination of scientific research documents, whether they are published or not. The documents may come from teaching and research institutions in France or abroad, or from public or private research centers.
L'archive ouverte pluridisciplinaire HAL, est destinée au dépôt et à la diffusion de documents scientifiques de niveau recherche, publiés ou non, émanant des établissements d'enseignement et de recherche français ou étrangers, des laboratoires publics ou privés. 


\section{REEDS Working Papers $_{\text {a }}$}

Cahiers de REEDS

\section{No.2012-07}

November 2012 / novembre 2012

\section{ECOLOGIE TERRITORIALE ET INDICATEURS POUR UN DÉVELOPPEMENT DURABLE DE LA MÉTROPOle PARISIENNE}

Johann AUDRAIN, Mateo CORDIER, Sylvie FAUCHEUX, Marie-Françoise GUYONNAUD, Isabelle NICOLAÏ, Martin O'CONNOR

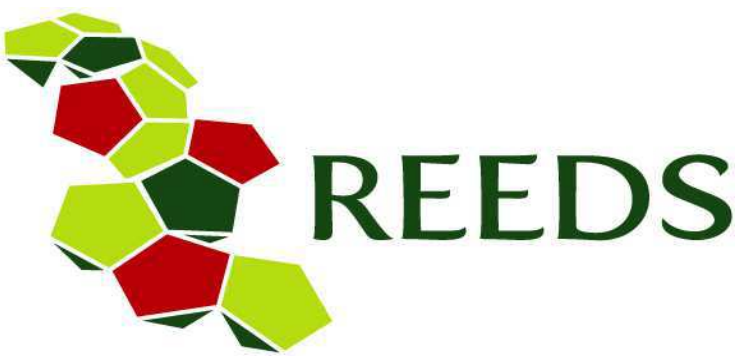

C Centre international " REEDS »

Université de Versailles Saint-Quentin-en-Yvelines Bâtiment " Aile Sud " UVSQ à la Bergerie Nationale

Parc du Château, 78514 Rambouillet cedex, France

Email : Secretariat.Reeds@uvsq.fr Website : www.reeds.uvsq.fr 



\title{
Ecologie Territoriale et Indicateurs pour un Développement Durable de la Métropole Parisienne
}

\section{Territorial Ecology and Indicators for Sustainable Development of the Metropolis of Paris}

\author{
Johann AUDRAIN \\ Chargé de recherche en écologie territoriale, FONDATERRA (Fondation Européenne pour des \\ Territoires Durables), France \\ Johann.Audrain@fondaterra.com
}

Mateo CORDIER

Maître de Conférences en Sciences économiques et Chercheur REEDS (Centre international de Recherches en Economie écologique, Eco-innovation et ingénierie du Développement Soutenable), Université de Versailles Saint-Quentin-en-Yvelines, France

Mateo.Cordier@reeds.uvsq.fr

Sylvie FAUCHEUX

Professeur en sciences économiques, REEDS \& Chaire ECONOVING, Université de Versailles SaintQuentin-en-Yvelines, France

Sylvie.Faucheux@uvsq.fr

Marie-Françoise GUYONNAUD

Directrice, FONDATERRA (Fondation Européenne pour des Territoires Durables), France Johann.Audrain@fondaterra.com

\section{Isabelle NICOLAÏ}

Professeur en sciences économiques, REEDS \& Chaire ECONOVING, Université de Versailles Saint-Quentin-en-Yvelines, France

Sylvie.Faucheux@uvsq.fr

\section{Martin O'CONNOR}

Professeur en sciences économiques et Directeur REEDS (Centre international de Recherches en Economie écologique, Eco-innovation et ingénierie du Développement Soutenable),

Université de Versailles Saint-Quentin-en-Yvelines, France

Martin.O-Connor@reeds.uvsq.fr

\footnotetext{
Note: Ce Cahier de REEDS est la version manuscrite d'un article qui sera publié en début 2013 dans le numéro spécial "Grand Paris" de la Revue d'Économie Régionale et Urbaine. Il reprend, mais avec un équilibre méthodologiquement sensiblement différent, des résultats d'analyse déjà présentés dans un précédent Cahier par Johann AUDRAIN, Mateo CORDIER, Sylvie FAUCHEUX, Marie-Françoise GUYONNAUD, Isabelle NICOLAÏ \& Martin O’CONNOR (2011), "La Ville et son Estuaire : Ecologie territoriale et Indicateurs pour un Développement Durable de la Métropole Parisienne", REEDS Working Papers / Cahiers de REEDS No.2011-11 (septembre 2011), UVSQ, Rambouillet.
} 


\section{Ecologie Territoriale et Indicateurs pour un Développement Durable de la Métropole Parisienne}

\section{Résumé}

Cet article traite de la dimension environnementale de la dynamique du «Grand Paris».Il explique comment une approche d'écologie territoriale permet d'aborder les questions de gouvernance pour la métropole Parisienne.Nous présentons, dans un premier temps, des indicateurs synthétiques comme l'«empreinte écologique» et l'«empreinte environnementale ", permettant d'appréhender l'ampleur de l'impact environnemental de la métropole.Ensuite, nous mettons en évidence, à partir d'une modélisation intégrée originale couplée à une analyse de scénario quantitative, le "métabolisme » du Grand Paris et, plus particulièrement, les impacts de l'activité économique du bassin de la Seine en amont sur l'écologie de l'estuaire de la Seine en aval.Les opportunités de politique territoriales suscitées par l'observation de la dégradation de l'estuaire sont démontrées.La nécessité d'accorder une place centrale à l'environnementdans toute vision stratégique des avenirs possibles de la mégapole parisienne devient alors explicite.

MoTs CLÉ : Développement durable territorial, Empreinte environnementale, Grand Paris, Modélisation intégrée, Services environnementaux

\section{Territorial Ecology and Indicators for Sustainable Development of the Metropolis of Paris}

\section{Abstract:}

This article treats the environmental dimension of the Greater Paris system.It shows how a territorial ecology approach permits the cogent investigation of governance principles and policy options for the Greater Paris region.First we present estimates of synthetic indicators such as the ecological footprint and the environmental footprint that allow us to quantify the scale of environmental impact of the mega-city.Then we consider, with an integrated modeling and scenario analysis, the "metabolism" of Greater Paris as illustrated by the impacts of economic activity in the Seine River catchment on the Seine Estuary downstream. This example highlights the important scope for territorial policies based on ecosystem quality considerations and, more widely, the need to accord a central place to the environmental dimension for strategic visions of possible futures of the Paris mega-city.

KEYWORDS: Environmental services, Environmental Footprint, Greater Paris, Integrated modelling, Sustainable territorial development.

JEL CaTegories: Q01 - Sustainable Development, Q57 - Ecological Economics, Q34 - Natural Resources and Domestic and International Conflicts 


\section{- 1 - \\ Introduction}

Cet article traite de la dimension environnementale de la dynamique du «Grand Paris », une métropole qui s'étend de la région Ile-de-France (dénommée par après "métropole parisienne ») jusqu'au Port du Havre dans le sous-bassin Seine aval (GRUMBACH, 2008). Le «Grand Paris » n'est pas seulement un projet urbanistique destiné à échafauder la métropole européenne du 21ème siècle, c'est déjà en partie une réalité : son organisation linéaire le long de la Seine favorise les déplacements, ses ports du Havre et de Rouen constituent des portes d'accès maritime aux marchandises vitales pour l'économie de la métropole parisienne, et ses territoires agricoles alimentent la population d'une métropole hautement tertiaire (GRUMBACH, 2008).

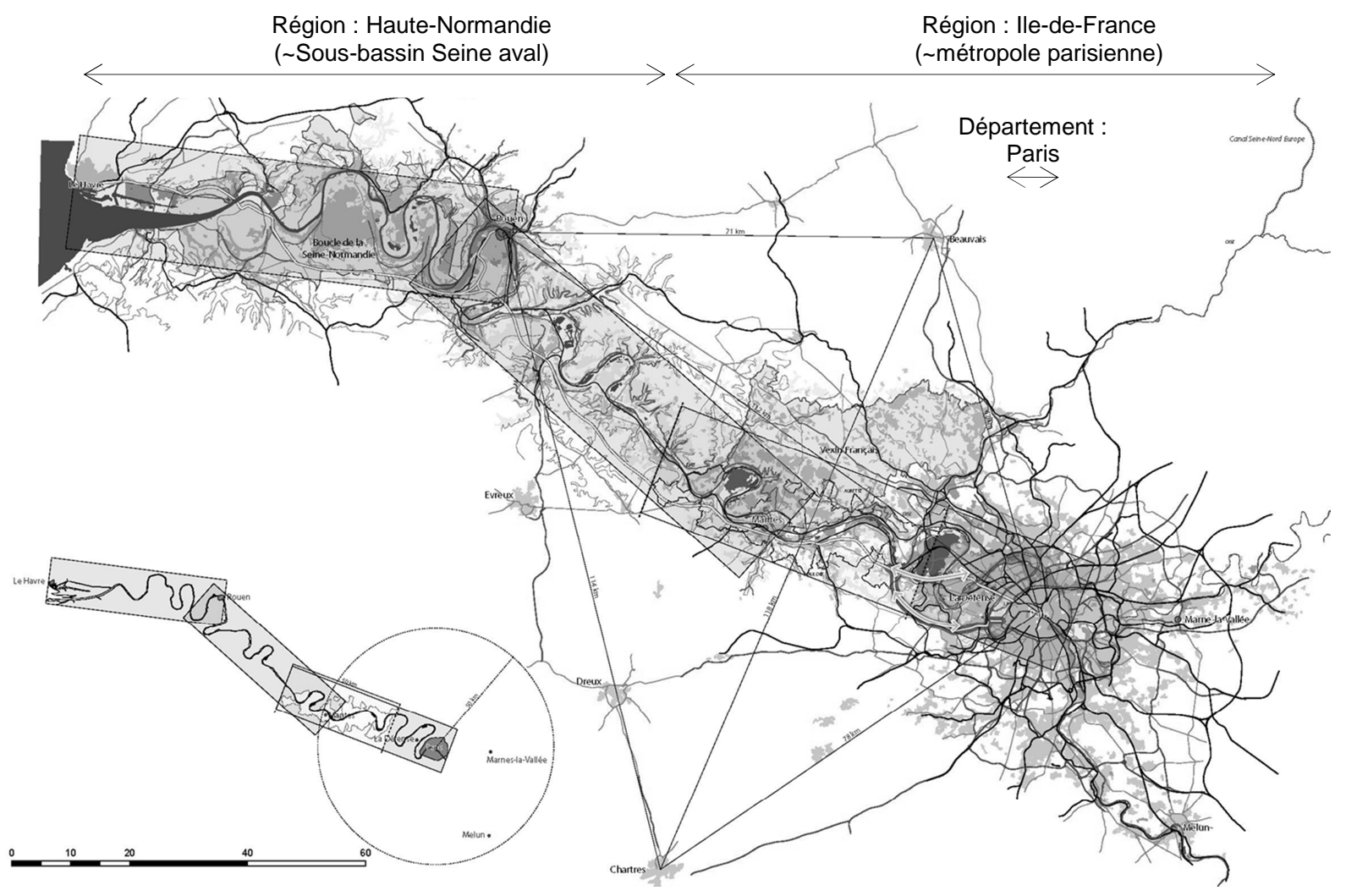

Figure 1. Le Grand Paris défini par Grumbach(2008) s'étend dela région Ile-de-France à la HauteNormandie.(C) ANTOINE GRUMBACH ET Assocí́s.

La ville métropole constitue l'une des principales sources d'émissions de polluants et de déchets divers. Elle contribue de manière importante et complexe aux problématiques environnementales et sociales des territoires de sa méga-région (ANDRES, BROCHET, 2010). L'armature de la ville métropole est composée de grands systèmes construits pour évacuer, distribuer, laisser circuler des flux de matières et d'énergie (réseaux de distribution d'eau et d'énergie, réseaux de collecte et de traitement des eaux usées et des déchets, voiries et réseaux de transport, systèmes d'information...). Ceux-ci assurent sa viabilité tout en organisant les 
conditions de sa consommation de ressources environnementales en qualité et en quantité. La méga-région inhérente à la ville métropole s'interprète alors comme un espace de flux (CASTELLS, 2001), un maillage en réseau d'activités, de ressources et d'individus.

Ainsi, le développement de la métropole parisienne ne se fait pas sans générer des problèmes environnementaux sur tout le territoire $\mathrm{du}$ 《 Grand Paris » et notamment au niveau de sa porte d'entrée maritime dans le sous-bassin Seine aval. Divers actifs environnementaux sont détruits ou dégradés alors qu'ils jouent un rôle important dans les activités économiques et autres activités humaines nécessaires au bien-être des individus. De ce point de vue, la question centrale de notre article est: comment une approche d'écologie territoriale peut irriguerles processus de gouvernance dans le but d'assurerque le développement économique et social du futur Grand Parisse fasse dans le respect des actifs environnementaux ? C'est la toute la question du développement durable qui consiste en la régulation de la sphère économique par rapport aux sphères sociale et environnementale afin d'assurer le respect simultané des critères de qualité et de performance propre à chacune des trois sphères.

Les outils d'écologie territoriale développés dans cet article sont susceptibles de retenir l'attention des décideurs et de servir les processus de gouvernance du Grand Paris pour une raison essentielle. Une famille de mesures reprise systématiquement par les dix agences d'architecture et d'urbanisme sélectionnées par le gouvernement en 2008 pour imaginer le futur visage du Grand Paris concerne la reconnexion entre les différents compartiments du territoire du Grand Paris. Ces mesure de reconnexion proposent par exemple derelier les centres aux périphéries par un réseau de transport en commun, d'assurer la liaison écologique entre les biotopes du territoire du Grand Paris par une trame verte et bleue, de relier les espaces verts aux espaces urbains pour assurer des services écosystémiques à la ville (ex. : régulation du climat, diminution du risque decrues par la rétention des eaux pluviales), etc. (WWF, 2009).Cependant, une reconnexion importante est négligée : la reconnexion entre le territoire terrestre et maritime du Grand Paris. Or les outils d'écologie territoriale développés dans cet article aident à comprendre le lien qui existe entre ces deux compartiments. Plus particulièrement, ces outils apportent un éclairage concernant l'impact de la consommation d'énergies fossilessur les habitats marins de l'estuaire de la Seine. Cet éclairage est important quand on sait queparmi les projets des agences d'architecture et d'urbanisme sélectionnés, nombreux sont ceux qui entérinent la multiplication des transports ${ }^{1}$ aériens (et des gaz à effet de serre émis par les avions) en renforçant les dessertes des aéroportsdu Grand Paris (WWF, 2009). Cela accentuerait la situation actuelle dans laquelle le transport aérien constitue déjà un des facteurs explicatif du niveau élevé de l'empreinte écologique des habitants de l'Ile-deFrance (voir Section 3.1). En outre, le transport aérien est fortement consommateur d'énergies fossiles (pétrole), ce qui en augmenterait les importations par voie maritime et risquerait d'accentuer les processus de dégradation des habitats marins de l'estuaire de la Seine. Cette dégradation est inquiétante quand on sait qu'elle vient renforcer la pression exercée par la pêche sur les stocks de poissons, pressions qui expliquent que l'estuaire de la Seine $^{2}$ ait vu sa biomasse totale des stocks de morues, de soles et de merlans diminuer de $43 \%$ entre 1978 et 2008 (ICES, accédé en 2009). Cette évolution n'est pas anecdotique. Le CIEM (ICES, 2008) estime que 32\% des stocks de poissons marins en Europe voient leur capacité de régénération mise en danger.

\footnotetext{
1 Il faut souligner cependant qu'en ce qui concernele transport, ces projets ne présentent pas que des effets négatifs au niveau environnemental. La plupart des agences sélectionnées suggèrent en parallèle le développement $\mathrm{du}$ transport en commun et du transport «doux» (pied, vélo, etc.) pour réduire l'impact environnemental des voitures individuelles (WWF, 2009).

2 Plus précisément la zone de pêche dans laquelle est située l'estuaire de la Seine (zone VIId du CIEM).
} 
Si les outils d'écologie territoriale apportent un éclairage pertinent sur la connexion entre les compartiments terrestres et maritimes du Grand Paris, il n'est cependant pas dit que de tels outils puissent retenir l'attention des politiques et des gestionnaires concernés. Cependant, ces outils sont très utiles pour apporter un éclairage scientifique aux approches de décision participative (voir Section 5.2 de cet article). Or la volonté affichée du projet de développement du Grand Paris est de se construire sur une logique de partenariat entre les acteurs institutionnels, sociaux et économiques. Bien que l'État joue un rôle essentiel, c'est aussi à travers la contractualisation et l'engagement réciproque de chacun (Etat, région,commune, investisseurs internationaux, petites et moyennes entreprises, associations de quartier,organismes de recherche, etc.) que ce grand projet commun se construit et se réalise(Ministère de l'Egalité des Territoires et du Logement, accédé en 2012). Cette logique participative de co-construction du Grand-Paris avec l'aide de tous les acteurs pourrait par conséquent se révéler propice au développement d'approches participatives d'aide à la décision et d'outils d'écologie territoriale destinés à éclairer la participation des acteurs.

Les outils d'écologie territoriale développés dans cet article permettent d'identifier les acteurs avantagés et désavantagés par un projet ou une mesure politique. Or c'est là un des objectifs principaux des approches participatives d'aide à la décision. En effet, les approches participative permettent de rendre les compromis plus transparents pour les décideurs et les acteurs, c'est-à-dire de bien identifier les acteurs et les ressources avantagés et désavantagés (Munda et al., 1994). C'est là tout l'intérêt des processus de décision participatifs car rendre les compromis clairs et apparents aux acteurs est nécessaire si l'on souhaite que les mesures politiques soient opérationnelles. Des réglementations et législations imposées par la force sans gagner en légitimité sociale ne fonctionnent pas bien. Quand elles ne sont pas contournées légalement par les acteurs, elles sont carrément enfreintes ou encore modifiées sans cesse par les groupes de pression importants.

Notre article est structuré comme suit. La Section 2 propose un cadrage méthodologique pour les analyses multi-échelles, à partir d'une structuration de la problématique environnementale en services écosystémiques et secteurs environnementaux. La Section 3 quantifie les besoins en ressources environnementales des activités économiques de la métropole parisienne à l'aide d'indicateurs synthétiques comme l' «empreinte écologique » et l' « empreinte environnementale». La Section 4 présente les grandes lignes d'une analyse complémentaire, aux échelles plus désagrégées. Elle traite du Grand Paris et de son estuaire à partir d'une modélisation économico-écologiques originale reposant sur des tables input-output (CORDIER et al., 2011) couplée à une analyse de scénario quantitative.Le modèle analyse le «métabolisme » du Grand Paris, et plus particulièrement les impacts de l'activité économique du bassin de la Seine en amont sur l'écologie de la Seine en aval. Les opportunités de politiques territoriales suscitées par l'observation de la dégradation de l'écologie de l'estuaire sont démontrées. La nécessité d'accorder une place centrale aux dimensions environnementales dans toute vision stratégique des avenirs possibles de la mégapole parisienne devient alors explicite. La Section 5 discute de la pertinence des deux outils, empreinte environnementale et modèle intégré, pour mieux articuler l'échelle spatiale globale et locale. Enfin, la Section 6 conclut sur l'apport des deux outils dans l'aide à la décision et la compréhension des interactions économie-écosystème. 


\section{- 2 - \\ Structurer l'interface Economie- Environnement: Eléments de Méthodologie}

Il existe de nombreuses typologies et méthodes de calculd'indicateurs concernant les différentes fonctions $\underline{d e}$ l'environnement pourl'économie, c'est-à-dire, des services environnementaux, dont la qualité ou la durabilité seraient mises en danger par des pressions. Nous exploitons dans cet article une méthodologie qui structure le problème sur base des deux concepts complémentaires: celui des «services environnementaux » (cf. Tableau 1) qui sont associés d'une manière ou d'une autre à des «actifs environnementaux » (cf. Tableau 2). Avec ce cadre méthodologique, il devient possible de décortiquer divers outils d'analyse et d'estimation d'indicateurs ainsi que d'en mesurer les points forts et les limites.

\subsection{Les Actifs environnementaux et les Services environnementaux}

Notre classement des «Actifs environnementaux » (cf. Tableau 2) est conforme aux conventions développées sur le plan international par le programme de réflexion et d'expérimentation qui promeut une nouvelle norme statistique pour le SEEA [System of integrated Economic and Environmental Accounts] sous le pilotage de l'UNCEEA. ${ }^{3}$

En ce qui concerne les "services environnementaux", nous adoptons des conventions de classement qui sont proches de celles retenues dans le MiLlenNiUM ECOSYSTEMASSESSMMENT (2005) et déjà proposées par DE GROOT et al. (2002).Le MiLLENNIUM ECOSYSTEMASSESSMENT (2005) définit quatre catégories de services écosystémiques : auto-entretien (ou «support»), régulation, approvisionnement en ressources, et services culturels. En ce qui nous concerne (FAUCHEUX, O'CONNOR, 1998; O'CONNOR, SCHOER, 2010), nous retenons une typologie ( $c f$. Tableau 1) qui est plus strictement "systémique" de caractère, notamment en insistant sur les processus cognitifs (par le biais d'un des cinq sens) avant de qualifier un service de "culturel" etc. Par conséquent, notre typologie regroupe les services écosystémiques en quatre catégories d'interactions, indépendamment du fait que ceux-ci peuvent avoir des finalités positives ou négatives pour les individus : 1) les structures assurant un service de support fonctionnel et de vie, 2) les flux de matière et d'énergie transitant depuisl'économie vers l'environnement, 3) les flux de matière et d'énergie transitant depuisl'environnement versl'économie et 4) les flux "presque" immatériels (dénommés "information" par DE GROOT et al., 2002) apportant des services cognitifs in situ captés par les cinq sens.

\footnotetext{
3 Voir http://unstats.un.org/unsd/envAccounting/ceea/. Y sont considérés comme «Actifs environnementaux », toutes les facettes ou «secteurs» de l'environnement (1) qui contribuent au bien-être individuel et collectif et, en particulier, à la productivité économique, et (2) dont les rôles économiques pour l'homme et son bien-être peuvent être modifiés de manière importante par ses propres actions. Le SEEA s'intéresse donc (1) aux contributions des actifs environnementaux par l'intermédiaire des services rendus aux processus économiques de production et de consommation, et (2) aux effets sur l'environnement des activités économiques. Ces interactions économie-environnement doivent être comptabilisées comme des «flux».On peut faire un rapprochement entre "actif" et la notion du "capital naturel"; en effet, un "capital" est un stock ou, plus généralement un fond entretenu de manière dynamique qui est une source de "services".
} 
Ces quatre types de "services" transitent aux interfaces entre des secteurs environnementaux $^{4}$ qui composent le milieu naturel (la biosphère, la lithosphère, l'hydrosphère et l'atmosphère; $c f$. Tableau 2) ainsi qu'entre des secteurs économiques.

Tableau 1.Typologiesystémique des services environnementaux (interactions entre économie et environnement)

Exemples :

\section{Services de support fonctionnel}

- Des sédiments marins (lithosphère sous-marine) qui constituent des habitats naturels pour les espèces aquatiques (biosphère).

- Les structures de biomasse (par exemple des feuilles des arbres) constituant autant d'habitats forestiers pour diverses espèces (au sein de la biosphère).

- Formes magmatiques dans la lithosphère assurant une certaine (in)stabilité des sols en surface pour les bâtiments, permettant la colonisation de milieux naturels par des infrastructures anthropiques

Flux de matière et d'énergie

\section{Flux Economie $\rightarrow$ Environnement}

Exemples: décharges des polluants dans le milieu naturel, etc.

3. Flux Environnement $\rightarrow$ Economie

Exemples: extraction de ressources naturelles

\section{Services cognitifs in situ}

Ressenti du milieu naturel par les cinq sens:
a) odorat
b) touché
c) vue
d) ouïe
e) goût

Exemples: appréciation du paysage, pollution sonore par un aéroport, calme des campagnes, etc.

Tableau 2. Structuration du milieu naturel en 4 grands secteurs et 7 sous-secteurs(O’CONNOR,SCHOER, 2010).

\begin{tabular}{ll}
\hline Biosphère : & Biodiversité marine, terrestre et volante, animale et végétale \\
\hline \multirow{2}{*}{ Lithosphère : } & Surface du sol (sols agricoles, sols forestiers...) \\
\cline { 2 - 2 } & Sous-sol (ressources minérales, ressources énergétiques, etc.) \\
\hline \multirow{2}{*}{ Hydrosphère : } & Eaux douces (souterraines et de surface) \\
\cline { 2 - 2 } & Eaux marines \\
\hline \multirow{2}{*}{ Atmosphère : } & Système climatique \\
\cline { 2 - 2 } & Qualité de l'air \\
\hline
\end{tabular}

Note: Etant donné que nous avons défini un nombre $n=7$ de sous-secteurs environnementaux (cf. Tableau 2), cela donnen $\times$ $(n-1)$ possibilités soit 42 possibilités d'échanges de matière et d'énergie entre ces secteurs. Or, nous avons défini 4 types d'interaction entre secteurs environnement et économie (cf. Tableau 1), ce qui donne au total un nombre de catégories d'interactions à considérer égalà $4 \times(n \times(n-1))$, soit un total de 168.Aucun outil d'analyse sauf des systèmes de comptabilité ne couvrira l'ensemble des catégories.

\subsection{Palier les limites de l'empreinte Ecologique par une analyse désagrégée}

L'une des approches communément mises en œuvre pour signaler la "pression" imposée par une activité économique sur son milieu biophysique (c'est-à-dire sur l'environnement) est l'empreinte écologique. Nous présenterons une application au territoire francilien dans la Section 3 suivante. Il s'agit d'un indicateur agrégé qui ramène la consommation des services environnementaux et des ressources naturelles à une seule unité : un équivalent de surface de

$4 \quad$ Si nous reprenons l'un des exemples du Tableau 1, l'accumulation d'énergie solaire par les végétaux dépend, parmi d'autres, d'un flux Environnement $\rightarrow$ Environnement qui a lieu entre deux secteurs environnementaux: le compartiment atmosphérique (le rayonnement solaire traverse l'atmosphère) et le compartiment de la biodiversité végétale (le rayonnement solaire est capté par les feuilles des arbres). 
terres nécessaires aux activités économiques de production et de consommation ${ }^{5}$. Le message d'un tel indicateur est simple à comprendre. Si les activités économiques consomment une quantité de ressources naturelles qui dépasse la quantité régénérée annuellement («biocapacité »), cela signifie que le capital naturel est en train d'être dilapidé.

Cependant, si une telle agrégation présente l'avantage de pouvoir sommer des services environnementaux très variés pour obtenir un score environnemental global, le désavantage provient du fait que toute agrégation implique une perte d'information et passe par des conventions toujours discutables et arbitraires. Un indicateur agrégé ne saurait refléter toutes les facettes de la complexité de l'environnement et des réalités importantes sont forcément occultées. Les problèmes environnementaux ne se résument pas à une consommation de surfaces de terrains. D'autres problématiques existent et nécessitent des unités diverses pour pouvoir être étudiées. Pensons par exemple aux problèmes d'approvisionnement en eau d'irrigation (mesurable en unités de volume), à la substitution des énergies fossiles par la biomasse végétale renouvelable (mesurable en kilowatt heures) ou encore à la qualité de l'eau qui affecte la vie aquatique (mesurable en milligrammes de polluants par litre d'eau).

C'est la raison pour laquelle l'empreinte écologique doit être complétée par d'autres indicateurs, plus désagrégés, capable d'exprimer diverses facettes de l'environnement. Les pressions environnementales sont extrêmement variées et distribuées spatialement de manière non homogène. La désagrégation doit donc s'exprimer sur plusieurs axes et notamment sur l'axe spatial.

Dans la Section 4, nous présentons quelques résultats d'un modèle économicoécologique régional (le modèle «Eco ») démontrant comment de tels modèles peuvent être utilisés pour offrir une vision spatiale désagrégée de certains aspects de l'empreinte écologique de la métropole parisienne. Ce modèle permet d'étudier les terres localisées dans le sous-bassin Seine Aval consommées par la population de la métropole parisienne pour assurer la production des produits économiques importés. Il permet de rendre compte que deux catégories de terres du sous-bassin Sein Aval sont nécessaires au fonctionnement de la métropole parisienne: les surfaces d'habitats marins de nourriceries de poissons et les surfaces d'eau pour le transport par bateau. Le problème mis en évidence provient du fait que la croissance du transport maritime est à l'origine de la croissance de l'activité portuaire dans le sous-bassin Seine Aval, croissance qui consomme les surfaces de nourriceries de poissons (DuCRotoy,DAuvin, 2008 ; Cuvillez et al.,2009 ; RochetTe et al., 2010 ; CoRDIER et al., 2011). Le modèle «Eco »permet de comprendre la relation entre la consommation de produits économiques par la métropole parisienne et la dégradation des surfaces de nourriceries dans le sous-bassin Seine aval ainsi que des populations de poissons marins.

Les deux échelles d'analyse et les différents niveaux d'agrégation se complètent. Sans ce complément d'information désagrégé sectoriellement et spatialement, l'empreinte écologique ne peut que servir de signal d'alarme un peu flou indiquant le degré de nonsoutenabilité de l'activité économique. Comme nous suggérons dans les Sections 5 et 6 de l'article, en complétant l'information apportée par l'empreinte écologique à l'aide d'outils désagrégés, il devient possible d'aider les décideurs à élaborer des mesures qui ciblent les secteurs économiques responsables de la dégradation des catégories spécifiques de services écologiques et dont les effets seront efficaces sur le plan écologique.

\footnotetext{
$5 \quad$ Au début du procédé de calcul de l'empreinte écologique, les quantités sont exprimées en unités diverses : tonnes de poissons, calories apportées par une tonne de maïs, hectares de blé ou de forêts, tonnes de $\mathrm{CO}_{2}$, kilowatts heures d'énergie nucléaire.Au final, elles sont toutes converties en une seule unité, des hectares globaux de terres.
} 


\section{- 3 - \\ Indicateurs synthétiques pour les interfaces Economie/Ecologie de la métropole parisienne}

\subsection{De l'empreinte écologique de la métropole parisienne...}

L'un des indicateurs communément proposé pour le diagnostic environnemental d'un territoire en termes de pressions et de services environnementaux, est l'empreinte écologique. Cet indicateur propose une estimation de la surface de «terres productives » qui seraient nécessaires pour supporter les activités humaines développées sur un territoire ou par une activité économique (WACKERNAGEL, REES, 1996).

L'empreinte écologique consiste, par exemple pour la consommation d'un carton de lait, à calculer la surface de prairies qui a été nécessaire pour nourrir les vaches laitières, la surface forestière requise pour la fabrication du carton, la surface des terres occupées par la ferme ainsi que la surface de terres dégradées par le transport et la vente finale du produit... Ensuite, au niveau d'un territoire, la surface totale nécessaire à la consommation de biens et de services des habitants est calculée pour une année donnée sur base de statistiques. Si cette surface dépasse les surfaces biologiquement productives existantes dans le territoire, les modes de consommation de ce territoire ne sont pas durables. Cela signifie que la consommation se réalise au détriment de la qualité environnementale du territoire et/ou grâce à l'importation de biens et de services d'autres territoires.

La notion d'empreinte écologique s'adresse à la question de quantification des pressions environnementales et des indicateurs synthétiques de la «pression » exercée par une activité économique par rapport à un territoire donné. Cette valeur est à comparer avec la surface réelle du territoire disponible afin d'obtenir un indice de la soutenabilité ou non des modes de vie sur celui-ci.

L'empreinte écologique de la métropole parisienne (couvrant l'ensemble du territoire de la Région d'Île-de-France) se définit ainsi par la surface de terres productives permettant la production des ressources consommées par les Franciliens et les activités de gestion des rejets générés (traitement des déchets, traitement de l'eau, etc.). Ce besoin en ressources naturelles traduit le mode de vie des habitants évalué à partir de leurs habitudes alimentaires, de leurs logements (superficie, mode de chauffage, etc.), de leurs déplacements (modes de transports, kilométrage, etc.), de la fabrication/fourniture, de leur consommation de biens et de services et de leur production de déchets.

Les calculs des «équivalences » en termes de terres productives, reposent nécessairement sur des conventions concernant les mesures de productivité des sols et les coefficients de conversion entre une ressource naturelle ou service environnemental et, son équivalent en terres. Dans la démarche prônée par WWF et le GFN (Global Footprint Network), il existe plusieurs sortes de sols : terres de cultures, pâturages, forêts, pêcheries, terrains construits (en supposant que les villes se sont le plus souvent construites sur des terres arables) et terrain carbone (correspondant aux forêts et océans nécessaires à l'assimilation du $\mathrm{CO}_{2}$ ). La méthode tient compte de la productivité biologique d'une surface de terre et non pas de sa seule superficie. Afin de pouvoir agréger ces différentes surfaces locales, de nature et de productivité biologique différentes, elles sont converties en une nouvelle unité, l'hectare global (hag), qui représente un hectare de terre ayant une productivité biologique égale à la moyenne mondiale (HaILset al., 2006 ; BoutEAU, GONDRAN, 2009 ; EWING et al., 2008). L'empreinte écologique est calculée de la façon suivante (Figure2) : 


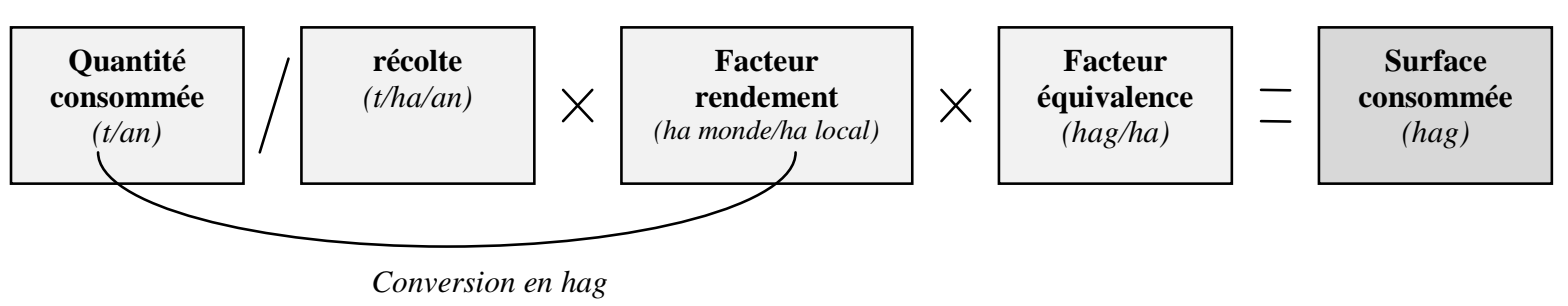

Figure 2. Méthode de calcul de l'empreinte écologique.

La conversion des hectares locaux en hectares globaux s'opère d'abord par l'utilisation de facteurs de rendements nationaux moyens permettant de prendre en considération les différences de productivité biologique entre pays. Sont utilisés ensuite des facteurs d'équivalence pour intégrer les différences de productivités moyennes mondiales selon les types de terres.

La question de la «soutenabilité » environnementale de l'activité économique d'un territoire, est posée en comparant cette empreinte écologique à la biocapacité du territoire. Cette dernière désigne la capacité à générer une offre continue en ressources renouvelables et à absorber les déchets découlant de la consommation de la population de ce territoire.

Une estimation de cet indicateur pour le territoire dela métropole parisienne a été communiquée par AUDRAIN et GUYONNAUD (2011), sur la base des données disponibles en 2010 (IAU, 2011). Cette analyse fournit les résultats suivants : l'empreinte écologique du francilien moyen est de 5,17 hag par an et par habitant, pour une biocapacité du territoire de 0,47hag. Plus de 50\% de l'empreinte écologique du Francilien est dû à l'usage de la «terre carbone ». L'empreinte écologique peut s'exprimer de deux manières, par type de consommation ( $c f$. Figure 3) ou par type d'usage des sols ( $c f$. Figure 4).

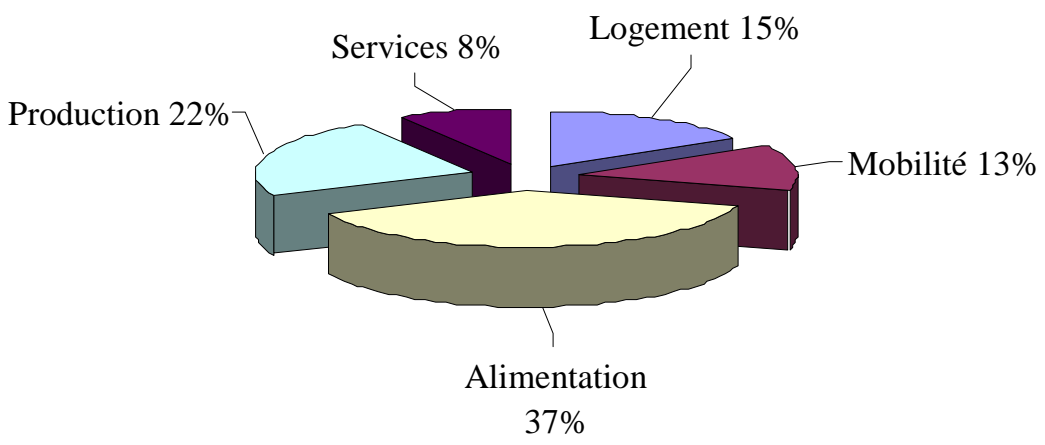

Figure 3. Empreinte écologique du territoire Ile-de-France par type de consommation en 2010 


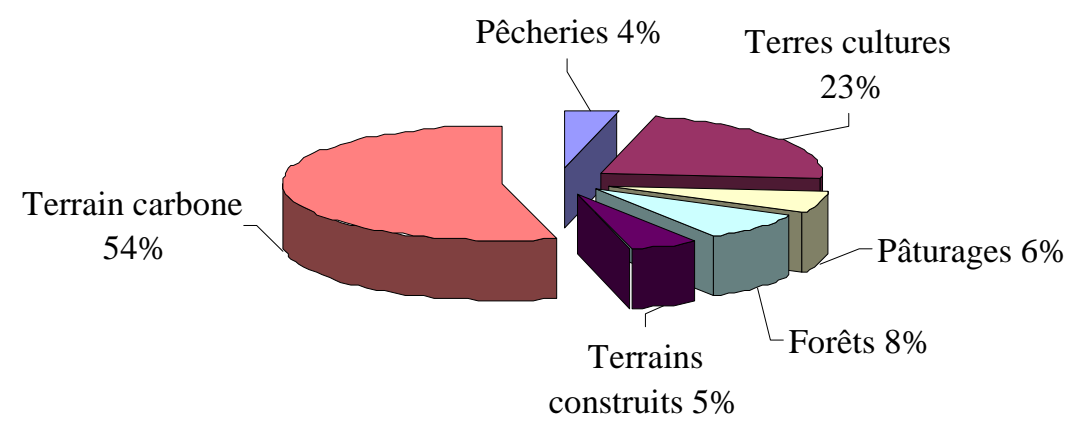

Figure 4. Empreinte écologique Ile-de-France par usage des surfaces en 2010

Plusieurs facteurs peuvent être avancés pour expliquer cette empreinte écologique relativement forte des habitants de la métropole parisienne :

- un niveau de vie plus élevé que la moyenne française (habitudes alimentaires, consommation de biens et services,...);

- les impacts du transport aérien avec la proximité des grandes plateformes aéroportuaires ;

- la concentration des nuisances et des pollutions liée à celle des activités et des mobilités professionnelles ;

- une population plus élevée que sur le reste de la France ;

- moins de terres productives en rapport avec d'autres territoires français.

L'espace nécessaire pour permettre aux Franciliens de satisfaire leurs besoins (les usages d'alimentation, logement, production, transports) s'avère par conséquent très supérieur aux limites administratives de Paris métropole. L'empreinte écologique de la métropole parisienne en 2010 est 11 fois supérieure à sa biocapacité. Autrement dit, si les habitants du monde entier "consommaient" comme les franciliens, l'humanité aurait besoin de 11 planètes Terre pour vivre. Ce type d'information est utile pour fournir une information sur le caractère de l'interdépendance des activités économiques d'un territoire et de l'environnement biophysiques alentour. Il permet de quantifier le degré de (in)soutenabilité environnementale de leur territoire.

\section{2....à son empreinte environnementale}

La question qui vient ensuite est la suivante : agir où et sur quoi ? Pour répondre à cette question, il est nécessaire d'avoir recours à des indicateurs désagrégés tant spatialement que sectoriellement. L'empreinte environnementale offre une réponse partielle à la question. Il s'agit d'une méthode de calcule très similaire à celle de l'empreinte écologique à la différence que l'empreinte du territoire est désagrégée par produits économiques. Cela permet d'identifier les produits qui contribuent le plus à l'empreinte écologique. Ce sont ces produits là que les mesures politiques devraient cibler prioritairement si l'on veut parvenir à la réduire efficacement. 
Les modalités de calcul conventionnel de l'empreinte écologique font en sorte que tous les impacts soient attribués à l'action de consommer des Franciliens. Il en résulte que la pression exercée par les secteurs d'activités de production économique sur les ressources d'un territoire n'apparaît pas. Or cette pression représente $22 \%$ de l'empreinte écologique de la métropole parisienne (Figure 3). La méthode, reposant sur l'approche entrées-sorties (inputoutput en anglais) développée par le NZCEE (New Zealand Centre for Ecological Economics) et adaptée par FONDaTERRA et son partenaire Market Economics à la France, permet d'évaluer les «terres nécessaires» pour satisfaire les besoins des différents secteurs d'activités d'un territoire, en l'occurrence celui de la métropole parisienne (AUDRAIN, GuYONNAUD, 2011).

Cette empreinte, dite " environnementale », aide à décrire et à quantifier la relation entre secteurs d'activités économiques et capital naturel afin d'élaborer des stratégies locales de développement économique et social et d'assurer la comparabilité des résultats entre les territoires sub-nationaux (MCDONALD, PATTERSON, 2004). L'approche entrées-sorties permet en effet la collecte de données autorisant une répartition détaillée, par secteurs d'activité, de l'empreinte des biens et services consommés dans un pays (BICKNELL et al.,1998; WIEDMANNet al., 2006). Elle aide à distinguer les intrants directs des intrants indirects clarifiant de ce fait ce qui appartient réellement à la demande finale (c.à.d. la consommation des franciliens dans notre cas) et en évitant les double-comptages.

Le calcul de l'empreinte environnementale repose sur l'ensemble des comptes économiques décrivant les transactions entre les divers secteurs économiques. Les calculs sont effectués pour les 36 secteurs de l'INSEE. En ce qui concerne les services et pressions environnementaux, il est possible d'obtenir jusqu'à douze types de terre avec l'hypothèse que ces dernières sont ou bien locales ou bien en dehors du territoire. Sont ainsi distinguées les terres horticoles et agricoles; terres santé et éducation; terres gouvernementales; terres mines et carrières ; terres industrielles; réseaux de transport et réseau d'adduction; terres commerciales ; terres loisirs ; terres résidentielles ; terres pêche ; terres énergies - permettant d'absorber le $\mathrm{CO}_{2}$ lié aux secteurs d'activités ; terres nucléaires.

Cette démarche a été appliquée à la Métropole parisienne (circonscrite au territoire de l'Ile-de-France) et désagrégée sur différents sous-territoires franciliens avec les résultats présentés à la Figure 5 (pour plus de détails, voir AUDRAIN, GUYONNAUD, 2011).

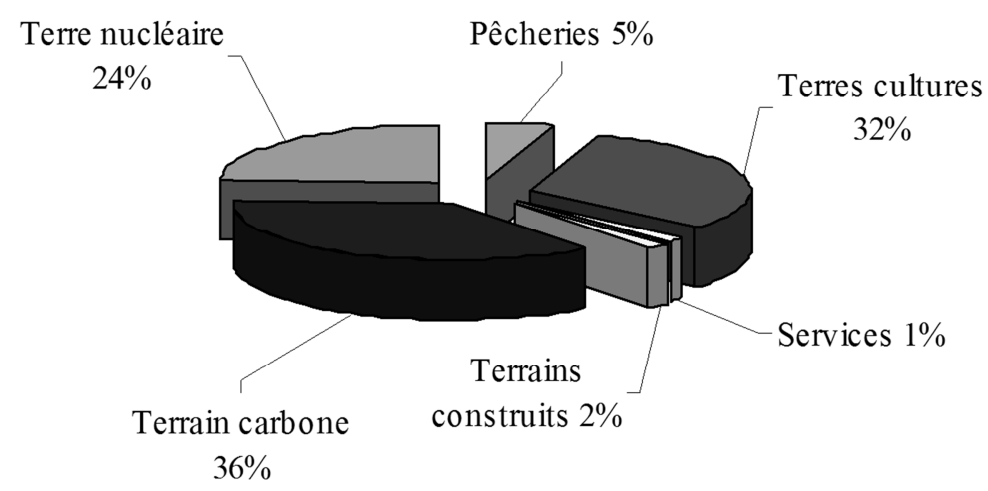

Figure 5. Empreinte environnementale de la région Ile-de-France par type de terres en 1999

En agrégeant ces résultats en 6 types de «terres » les résultats obtenus sont proches de ceux calculés par l'IAU en 2010. L'empreinte environnementale non seulement, représente la 
quantité d'hectares globaux nécessaires à chaque type de terres utilisées par les secteurs d'activités ; mais elle indique aussi l'origine de chaque type de terres, provenant du territoire (surfaces de terres du territoire nécessaires à la production des biens et les services du territoire) ou importée d'autres territoires (surfaces de terres associées à la production de biens et de services importés par le territoire).

Le Tableau 3 représente la quantité d'hectares globaux nécessaires à chaque type de terres inclus dans l'empreinte environnementale.

Tableau-3: Empreinte environnementale d'Ile-de-France(hag) par type de terres, 1999

\begin{tabular}{|c|c|c|c|c|c|}
\hline Usages des terres & $\begin{array}{c}\text { Terres au } \\
\text { sein du } \\
\text { territoire } \\
\text { hag } \\
\end{array}$ & $\begin{array}{c}\text { Terres en } \\
\text { dehors du } \\
\text { territoire } \\
\text { hag } \\
\end{array}$ & $\begin{array}{c}\text { Surface } \\
\text { totale de } \\
\text { terres } \\
\text { hag } \\
\end{array}$ & $\begin{array}{c}\begin{array}{c}\text { Surface } \\
\text { totale de } \\
\text { terres }\end{array} \\
\text { hag/habitant }\end{array}$ & $\begin{array}{c}\begin{array}{c}\text { Surface } \\
\text { totale de } \\
\text { terres }\end{array} \\
\% \text { du total } \\
\end{array}$ \\
\hline Terres horticoles et agricoles & 3055200 & 14222600 & 17277800 & 1,6 & $31,8 \%$ \\
\hline Terres santé et éducation & 51600 & 11500 & 63100 & 0,0 & $0,1 \%$ \\
\hline Terres gouvernementales & 63700 & 11200 & 74900 & 0,0 & $0,1 \%$ \\
\hline Terres mines et carrières & 14300 & 70200 & 84500 & 0,0 & $0,2 \%$ \\
\hline Terres industrielles & 68300 & 58000 & 126300 & 0,0 & $0,2 \%$ \\
\hline Réseaux de transport et réseau d'adduction & 99800 & 58900 & 158700 & 0,0 & $0,3 \%$ \\
\hline Terres commerciales & 48300 & 12700 & 61000 & 0,0 & $0,1 \%$ \\
\hline Terres loisirs & 70100 & 14700 & 84800 & 0,0 & $0,2 \%$ \\
\hline Terres résidentielles & 929200 & 0 & 929200 & 0,1 & $1,7 \%$ \\
\hline Terres pêche & 2699700 & 0 & 2699700 & 0,2 & $5,0 \%$ \\
\hline $\begin{array}{l}\text { Terres énergies }-\mathrm{CO}_{2} \text { provenant } \\
\text { combustibles fossiles }\end{array}$ & 11557900 & 7967600 & 19525500 & 1,8 & $35,9 \%$ \\
\hline Terres nucléaires & 13282400 & 0 & 13282400 & 1,2 & $24,4 \%$ \\
\hline Total & 31940500 & 22427400 & 54367900 & 4,9 & $100,0 \%$ \\
\hline
\end{tabular}

SOURCE: FONDATERRA (2010, p. 209).

Le Tableau 4 illustre ensuite, la quantité d'hectares globaux nécessaires pour produire les biens et services consommés par les ménages du territoire étudié.

Les composantes principales de l'empreinte environnementale sont les postes «énergie » et «alimentaire », comme l'avait mis en évidence le diagnostic empreinte écologique dans la section précédente. L'empreinte environnementale permet d'éclairer le diagnostic environnemental en termes de consommations de terres issues du territoire étudié et de celles provenant d'autres territoires par tel ou tel secteur d'activités. Certains de ces secteurs s'avèrent fort consommateurs d'empreinte environnementale soit en local, soit en terres extérieures ou encore en cumulant les deux. Face à cette évaluation de consommation de terres pour produire des biens et services, le territoire peut mettre en regard les apports locaux liés au développement économique du secteur d'activité en termes d'emplois, de rentabilité financière, de renommée internationale, de réputation scientifique. Ces informations relatives à l'empreinte environnementale éclairent les choix des collectivités territoriales en termes de stratégie de développement et d'aide à la décision pour les aménagements existants et futurs. 
Tableau 4 : Empreinte environnementale d'Ile-de-France(hag) par produit économique, 1999

\begin{tabular}{|c|c|c|c|c|c|}
\hline Produits économiques & $\begin{array}{c}\text { Terres au } \\
\text { sein du } \\
\text { territoire } \\
\text { hag } \\
\end{array}$ & $\begin{array}{c}\text { Terres en } \\
\text { dehors du } \\
\text { territoire }\end{array}$ & $\begin{array}{c}\text { Surface } \\
\text { totale de } \\
\text { terres } \\
\text { hag } \\
\end{array}$ & $\begin{array}{c}\begin{array}{c}\text { Surface } \\
\text { totale de } \\
\text { terres }\end{array} \\
\text { hag/habitant }\end{array}$ & $\begin{array}{c}\begin{array}{c}\text { Surface } \\
\text { totale de } \\
\text { terres }\end{array} \\
\% \text { du total } \\
\end{array}$ \\
\hline Alimentation & 1803200 & 10006900 & 11810100 & 1,1 & $21,7 \%$ \\
\hline $\begin{array}{l}\text { Autres produits primaires(inclut les } \\
\text { carburants issus du raffinage du pétrole) }\end{array}$ & 3717400 & 1535400 & 5252800 & 0,5 & $9,7 \%$ \\
\hline Vêtements et textiles & 82900 & 379700 & 462500 & 0,0 & $0,9 \%$ \\
\hline Papier, imprimerie et édition & 78600 & 147800 & 226400 & 0,0 & $0,4 \%$ \\
\hline $\begin{array}{l}\text { Produits chimiques et dérivés du pétrole (à } \\
\text { l'exclusion des carburants) }\end{array}$ & 565600 & 353500 & 919200 & 0,1 & $1,7 \%$ \\
\hline Machineries, équipements et appareils & 415400 & 914600 & 1330000 & 0,1 & $2,4 \%$ \\
\hline Autres produits manufacturés & 569600 & 940500 & 1510100 & 0,1 & $2,8 \%$ \\
\hline Electricité, gaz et eau & 6446300 & 152700 & 6598900 & 0,6 & $12,1 \%$ \\
\hline Construction & 1459400 & 1183700 & 2643100 & 0,2 & $4,9 \%$ \\
\hline Transport & 822600 & 172400 & 995100 & 0,1 & $1,8 \%$ \\
\hline Commerce de gros et de détail & 1035400 & 2700 & 1038100 & 0,1 & $1,9 \%$ \\
\hline Services aux entreprises & 1074800 & 295100 & 1370000 & 0,1 & $2,5 \%$ \\
\hline Logement, restauration et bars/cafés & 693100 & 1578900 & 2272000 & 0,2 & $4,2 \%$ \\
\hline Culture, loisirs et services d'intérêt général & 626300 & 118100 & 744400 & 0,1 & $1,4 \%$ \\
\hline Santé et éducation & 1596300 & 1169500 & 2765800 & 0,3 & $5,1 \%$ \\
\hline $\begin{array}{l}\text { Services gouvernementaux: administration } \\
\text { publique }\end{array}$ & 1195500 & 286000 & 1481500 & 0,1 & $2,7 \%$ \\
\hline Services pour les ménages ${ }^{8}$ & 9757900 & 3190000 & 12947800 & 1,2 & $23,8 \%$ \\
\hline Total & 31940300 & 22427500 & 54367800 & 4,9 & $100,0 \%$ \\
\hline
\end{tabular}

SOURCE: FONDATERRA (2010, p. 209).

FONDaTERRA et son partenaire Market Economics Ltd ont ainsi pu proposer un diagnostic d'«efficiencedes terres » sur des territoires donnés d'Ile-de-France. L'efficience des terres représente la production générée par la terre (évaluée en millions d'euros) rapportée à la superficie utilisée (mesurée en hag). Les calculs d'empreinte environnementale ont été désagrégés à différentes échelles dont celle du département des Yvelines et de l'Opération d'Intérêt National (OIN) Seine Aval. Ce calcul permet de définir l'efficience des principaux secteurs d'activités économiques (Tableau 5, sur la page suivante).

Sur ce territoire, en 2006, un hectare consacré à la production agricole produisait une valeur économique11500 fois plus faible qu'un hectare consacré à la production automobile.

6 La catégorie «Autres produits primaires » couvre les produits agricoles et horticoles, les minerais sableux, pierreux, métalliques et énergétiques (uranium, gaz naturel, charbon, pétrole, etc.), les produits pétroliers raffinés (carburants), la transformation de l'uranium avant usage (dans le centrales nucléaires notamment).

7 La catégorie des produits pétroliers et chimiques concernent par exemple les plastiques dérivés du pétrole mais ils ne couvrent pas les carburants fabriqués dans les raffineries de pétrole. Ceux-ci sont déjà inclus dans «Autres produits primaires ».

Exemples de services pour les ménages : blanchisseries, salons de coiffure, pompes funèbres, femme de ménage. 
Tableau 5. Efficience des terrespour lesprincipaux secteurs économiques des Yvelines et OIN Seine aval en 2006 (tous les secteurs sauf l'Extraction) et d'Ile-de-France en 1999 (secteurs d'Extraction).

\begin{tabular}{|c|c|c|c|c|c|c|}
\hline & Agriculture & $\begin{array}{c}\text { Extraction et } \\
\text { traitement des } \\
\text { ressources } \\
\text { énergétiques }\end{array}$ & $\begin{array}{c}\text { Extraction de } \\
\text { sable, pierres et } \\
\text { minerais } \\
\text { métalliques }\end{array}$ & Industrie & Automobile & Services \\
\hline $\begin{array}{c}\text { Efficience } \\
\text { (euros/hag) }\end{array}$ & 558 & 2614650 & 253354 & 1372804 & 6441873 & 305946 \\
\hline
\end{tabular}

AUDRAIN, GUYONNAUD (2011) et calculs propres à partir des données Excel transmises par e-mail par les auteurs en octobre 2011.

L'empreinte environnementale calcule les équivalents en «terres » nécessaires pour couvrir les besoins des différents secteurs d'activités d'un territoire (donc la consommation d'un territoire). En désagrégeant ce territoire, il est possible d'obtenir des évaluations de l'utilisation des terres par secteurs d'activité présents sur ce territoire et d'ouvrir ainsi la réflexion sur la nature des activités les plus adaptées au territoire. En comparant l'efficacité des terres et les modes de consommation, l'empreinte environnementale devient alors un outil de diagnostic environnemental et d'aide à la décision en matière de choix d'aménagement de la métropole parisienne, complétant utilement d'autres outils plus traditionnels. ${ }^{10}$

Il s'agit d'un outil efficace pour montrer combien les différents types d'activités humaines d'un territoire exigent, chacun, une disponibilité de terres (ici, terres biologiquement productives). Toutes ces demandes étant en compétition pour l'espace et les ressources écologiques, les analyses en termes d'empreinte environnementale permettent de cadrer des stratégies locales pour réduire l'impact des modes de vie.

Cependant, l'empreinte environnementale désagrégée par produits économiques ne résout pas toutes les limites de l'empreinte écologique. Elle ne spécifie pas quel type de mesure prendre concernant ces produits ni sur quel territoire les prendre. Or nous avons vu que dans le cas de la métropole parisienne, environ $41 \%$ de son empreinte environnementale est due à l'importation de biens et services fabriqués sur des surfaces de terres localisées dans d'autres régions de France et du monde (calculé sur base duTableau 4) ${ }^{11}$. Des outils désagrégés spatialement doivent compléter les informations données par les empreintes environnementales désagrégées sectoriellement (ou par produits économiques). C'est l'objet dumodèle économico-écologiques régional «Eco » développé par CORDIER (2011) et présenté à la Section suivante.

Le secteur de l'extraction et du traitement des ressources énergétiques couvre : l'extraction de charbon, gaz, pétrole et uranium ainsi que la raffinage du pétrole et le traitement du charbon et de l'uranium.

Voir DECAMPS et VICARD (2010) pour une analyse comparative des indicateurs de développement durable territoriaux.

${ }^{11}(41 \%=100 * 22427500$ hag / 54367800 hag $)$ 


\section{-4 - \\ Articulation des échelles d'analyse : passage d'une échelle agrégée à une échelle désagrégée}

Les empreintes agrègent une grande quantité d'informations dans un indicateur unique et rapidement compréhensible, ce qui facilite la promotion des concepts de durabilité et de dommages écologiques dans les processus de décisions politiques. Toutefois, ces indicateurs ne sont pas exempts d'inconvénients. Tout d'abord, et la partie précédente l'a montré, il existe des divergences quant aux méthodes de calcul qui leur sont sous-jacentes. Le manque de standardisation ne favorise pas la comparaison d'empreintes écologiques ou environnementales entre régions ou pays. Ensuite, renvoyer toute question de "durabilité » environnementale à une question de surface de «terres» ou à des équivalents, semble artificiel et excessif. La simplicité du concept se trouve, en quelque sorte, diluée dans la nécessité de préciser plusieurs catégories de «terres» en adéquation avec des catégories différentes de pressions ou de services environnementaux.

Pour une analyse plus fine des enjeux environnementaux et des options de stratégie industrielle, territoriale et politique, il parait indispensable d'intégrer la «spécificité » des actifs et des services environnementaux. Une démarche, allant dans cette direction et complémentaire à l'ambition synthétique de l'empreinte écologique (ou environnementale), est présentée dans ce qui suit. Elle propose une analyse intégrée des impacts de l'activité économique du Grand Paris sur des écosystèmes et des services environnementaux de son estuaire, à savoir l'Estuaire de la Seinequi est localisé dans le sous-bassin Seine aval (Figure 1).

\subsection{Un modèle intégré appliqué au Grand Paris et à son estuaire}

En termes biophysiques, le «métabolisme » du territoire de l'estuaire de la Seine peut être représenté de manière simplifiée par deux systèmes, l'environnement et l'économie, à l'interface desquels de la matière et de l'énergie transitent. Parmi les outils d'une telle représentation, on trouve notamment des modèles économico-écologiques reposant sur des tables input-output (DALY, 1968 ; VICTOR, 1972b, MCDONALD, 2005). Lorsque la matière et l'énergie passent du système économique vers l'écosystème, on parle d'émissions polluantes et de déchets ou encore de colonisations d'infrastructures urbaines et industrielles sur des zones d'habitats naturels. Lorsqu'elles traversent l'écosystème en direction du système économique, on parle d'extraction de ressources naturelles, comme la pêche ou l'extraction de granulats. C'est finalement l'ensemble du système de production économique et de consommation qui peut se résumer dans ces transferts d'interface dans la mesure où toute consommation de bien et service a nécessité, à un moment ou un autre, la consommation d'une ressource naturelle, qu'elle soit matérielle ou énergétique (FAUCHEUX, O'CONNOR, 1998 ; HAAKE, 2000).

CORDIER (2011) a conçu un modèle économico-écologique de ce type dénommé cidessous modèle «Eco ». Celui-ci simule l'interaction entre l'écosystème de l'estuaire de la Seine et son système économique. Les activités portuaires (ports du Havre et de Rouen) constituent un facteur moteur $\mathrm{du}$ fonctionnement du système économique dont le 
développement est largement entraîné par la consommation du reste de la France.La région Haute-Normandie (qui inclut l'estuaire de la Seine) assure 32,3\% des expéditions nationales (exportations interrégionales) en produits pétroliers raffinés et fournit 10,3\% de l'électricité produite en France (LEROND, 2006). Une partie très importante de ces flux est évidemment destinée à la métropole parisienne.

Pour donner un ordre de grandeur de l'effet de la métropole parisienne sur le sousbassin Seine Aval, prenons les exportations interrégionales du sous-bassin (plus exactement de la Haute-Normandie): $22 \%$ de ces biens étaient destinés à l'Ile-de-France en 2006. C'est conséquent quand on sait que les exportations vers les autres régions sont généralement inférieures à $6 \%$ du total exporté par la Haute-Normandie en France (calculs propres basés sur les données de flux interrégionaux du MEEDDAT, 2009).

Alors que l'estuaire procure des services écosystémiques intermédiaires essentiels au bon fonctionnement global de l'écosystème (ex. :habitats de nourriceries, stockage et détoxification des polluants), il offre également des services écosystémiques finaux attirant de nombreuses activités industrielles (ex. : approvisionnement en eau pour le transport maritime). Or, ces dernières génèrent des nuisances entravant les services écosystémiques intermédiaires. A titre d'illustration, depuis 1834 , les surfaces de nourriceries subissent une destruction lente et progressive en raison du processus d'anthropisation croissante de l'estuaire: développement du port de Rouen et du Havre, construction du pont de Normandie et de digues pour la navigation maritime (DUCRotoy, DAUvin, 2008 ; CuVILlEZ et al., 2009). Entre 1834 et 2004, 33\% de la surface de nourriceries potentielles ont été transformés en zone terrestre (ROCHETTE et al., 2010). La surface de nourriceries à haute capacité d'accueil (> 45 individus de juvéniles de soles par $\mathrm{km}^{2}$ ) s'élevait à $182 \mathrm{~km}^{2}$ en 1834 alors qu'en 2004, elle n'atteignait plus que $112 \mathrm{~km}^{2}$ (CORDIER et al., 2011).

Le modèle en question engage une analyse de processus écologique analogue à celle de processus économique dans le système industriel. Il procède en trois étapes : (a) identifier les systèmes et les services écosystémiques en jeu et les chaînes de causalité auxquelles ils participent (voir FISHER et al.,2009); (b) quantifier les paramètres physiques impliqués dans les flux d'interface économie/environnement liés à ces services écosystémiques en vue de les insérer dans un modèle économico-écologique ; et (c) quantifier les interrelations entre les différentes étapes de la chaîne de causalité. L'ensemble de ces étapes nécessite un long déroulement (détaillé dans CORDIER, 2011) et ne sont synthétisés ici que quelques grandes lignes (Figure 6).

Figure 6 : Exemple de services écosystémiques découpés sur base d'une version adaptée de la chaîne de causalité de FISHER et al. (2009).

\begin{tabular}{|c|c|c|c|}
\hline \multicolumn{2}{c|}{$\begin{array}{c}\text { Services intermédiaires } \\
\text { (structures et processus) }\end{array}$} & Services finaux \\
De premier ordre : & $\begin{array}{c}\text { Pe second ordre : } \\
\text { sédimentaires permettant } \\
\text { l'accumulation de sédiments } \\
\text { fins à faible profondeur }\end{array}$
\end{tabular}

Les services finaux sont plus directement liés aux usages anthropiques et au bien-être puisque ce sont eux qui génèrent les bénéfices dont profitent les individus (CORDIER, 2012). 
Par exemple, les processus hydro-sédimentaires constituent un service écosystémique intermédiaire de premier ordre qui permet l'accumulation de sédiments fins à faible profondeur formant ainsi une structure physique sur laquelle un processus écologique de formation de vasière peut se dérouler. Les vasières fournissent à leur tour un service intermédiaire de second ordre en offrant un habitat de nourricerie essentiel pour les jeunes alevins qui viennent s'y nourrir et se développer. La concentration d'alevins dans ces habitats procure un service final d'approvisionnement en ressources halieutiques dont l'homme tire un bénéfice en vendant et en consommant ces ressources. Cela n'est cependant possible que grâce à la transformation des services finaux (utilité potentielle) en bénéfices (utilité effective). En général, les services finaux peuvent être transformés en bénéficesuniquement par l'intermédiaire d'un savoir-faire (en techniques de pêche) et d'équipements spécialisés (filets, chalutiers, etc.).

Après l'identification des catégories clés pour la modélisation, la seconde étape est celle de la quantification des paramètres physiques liés aux flux de matière et d'énergie transitant à l'interface économie/environnement. En reprenant l'exemple de l'approvisionnement en ressources halieutiques (poissons) de la Figure 3 pour chacune des quatre catégories de services écosystémiques, des flux de matières peuvent être identifiés. Pour les bénéfices, il s'agit de tonnes de poissons pêchés par an et de valeur ajoutée produite annuellement par le secteur de la pêche exprimée en euros. En ce qui concerne la matière impliquée dans le service final de développement de jeunes alevins, sa mesure peut s'effectuer en nombre d'alevins par hectares de nourricerie ou en tonnes d'alevins par an. Le service intermédiaire de second ordre lié à la formation de vasières,peut être évalué par le nombre d'hectares de vasières existantes. Le service intermédiaire de premier ordre, relatif aux processus hydrosédimentaires assurant la formation des vasières par l'apport de sédiments fins à faible profondeur,peut être mesuré en tonnes de sédiments marins.

\begin{tabular}{|c|c|c|c|}
\hline & & \multicolumn{2}{|c|}{ DESTINATION } \\
\hline & & Economie & Ecosystème \\
\hline \multirow{2}{*}{ 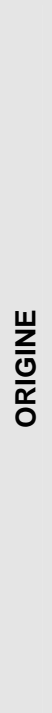 } & 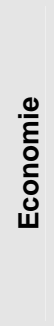 & $\begin{array}{l}\text { 1. Echange de biens et services économiques sur le } \\
\text { marché } \\
\text { Ex.: croissance du transport maritime et extension } \\
\text { subséquente des activités portuaires (mesurée par la } \\
\text { valeur ajoutée en unités monétaires) }\end{array}$ & $\begin{array}{l}\text { 2. Services écosystémiques intermédiaires de } \\
\text { premier ordre } \\
\text { Ex.: destruction des habitats de nourriceries pour } \\
\text { jeunes alevins (service intermédiaire) causée par } \\
\text { l'extension des infrastructures portuaires et la } \\
\text { modification subséquente des dynamiques hydro- } \\
\text { sédimentaires (mesurée en hectares de } \\
\text { nourriceries détruits) }\end{array}$ \\
\hline & 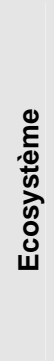 & $\begin{array}{l}\text { 4. Services écosystémiques finaux et bénéfices } \\
\text { Ex. : la diminution du stock total de nourriceries génère } \\
\text { une baisse du nombre d'alevins arrivant à l'âge adulte et } \\
\text { in fine, une diminution de la taille de la population de } \\
\text { poissons (service final) (mesurée en tonnes de } \\
\text { poissons). Cela conduit à une baisse du nombre de } \\
\text { captures de pêches (bénéfices) (mesurés en tonnes } \\
\text { pêchées et en valeur ajoutée du secteur de la pêche en } \\
\text { unités monétaires). }\end{array}$ & $\begin{array}{l}\text { 3. Services écosystémiques intermédiaires de } \\
\text { second ordre } \\
\text { Ex. : état du stock total de nourriceries existant } \\
\text { utilisé par les jeunes alevins(mesuré en hectares } \\
\text { de nourriceries restants) }\end{array}$ \\
\hline
\end{tabular}

Figure7 : Flux de matières et d'énergies aux interfaces entre le système économique et l'écosystème illustré par le service final d'approvisionnement en poissons. 
La Figure 7 illustre de manière simplifiée un modèle conceptuel d'un anthropoécosystème sous la forme de deux systèmes : un écosystème et un système économique à l'interface desquels de la matière et de l'énergie transitent. Ce schéma a été construit par CORDIER (2011) et est inspiré des modèles input-output environnementaux de DALY (1968), ISARD (1968), ViCTOR (1972a) et plus récemment, CARPENTIER (1994), JiN et al. (2003), MCDONALD (2005) et ZHANG $\mathrm{t}$ al.(2008). Il est composé de quatre interfaces (les quatre quadrants de la Figure 7) représentant les flux internes à l'économie (quadrant 1), les flux transitant depuis l'économie vers l'écosystème (quadrant 2), les flux internes à l'écosystème (quadrant 3), et les flux passant de l'écosystème vers l'économie (quadrant 4).

Une fois les flux d'interface identifiés et quantifiés, une dernière étape doit être effectuée. Il s'agit de quantifier les interrelations entre les différents flux. Il faut, par exemple, pouvoir calculer la relation mathématique liant l'évolution du transport maritime et des activités portuaires (quadrant 1) à la colonisation des habitats de nourriceries par les infrastructures portuaires (quadrant 2). Il en va de même pour la relation mathématique mettant en relation l'évolution du stock de nourriceries (quadrant 3) et les captures de pêche (quadrant 4). Si ces relations sont présumées linéaires, les modèles économico-écologiques de type input-output offrent une solution relativement simple par le calcul de coefficients techniques d'outputs écologiques (pour plus de détails, voir, Miller et Blair, 2009; McDonald, 2005). En revanche lorsque ces relations ne sont pas linéaires, il est nécessaire de recourir à des équations exogènes au modèle input-output. C'est le cas de la relation mathématique qui lie la colonisation des habitats de nourriceries par les infrastructures portuaires (quadrant 2) au stock de nourriceries (quadrant 3).

Pour le système économique, le modèle input-output repose sur les tables supply-use (aussi appelées commodity by industry) représentant la manière dont les industries échangent entre elles des matières premières, des produits semi-finis et des services pour fournir la demande finale en produits finis consommés par les ménages.

\subsection{Analyse comparative de scénarios}

Ce modèle économico-écologique a été appliqué au cas d'un scénario de restauration des nourriceries de l'estuaire de la Seine utilisées comme habitat marin par les juvéniles de soles pour se nourrir et se développer. Sur base de l'observation des pertes ensurfaces de nourriceries depuis 1834, trois scénarios ont été élaborés puis simulés dans le modèle économico-écologique.

(1) Un scénario de référence « business as usual » (BAU) qui représente l'écosystème et l'économie en 2015 tel qu'ils auraient évolués à partir de 2004 si la tendance actuelle se prolongeait et qu'aucune mesure de restauration des nourriceries n'était entreprise.

(2) Un scénario de restauration de $25 \%$ des surfaces de nourriceries sableuses à haute densité de poissons détruites entre 1834 et 2004. Le scénario est échelonné sur une période s'étalant entre 2004 et 2015 . Cela revient à restaurer $2,2 \mathrm{~km}^{2} /$ an pendant 11 ans, soit une surface totale restaurée de $24,38 \mathrm{~km}^{2}$. Le coût de restauration annuel s'élève à 386 millions $€_{2007} /$ an. Dans ce scénario, les coûts de restauration sont pris en charge intégralement par les ports (responsables directs de la destruction des habitats).

(3) Un scénario de restauration identique au précédent mais avec un partage des coûts entre tous les secteurs primaires et secondaires qui bénéficient des services portuaires pour le transport par voie d'eau de leurs marchandises (responsables indirects de la destruction des habitats). L'allocation des coûts de restauration devrait en pratique être débattue et décidée collégialement par les acteurs de l'estuaire de la Seine s'il s'avère que ce scénario de 
restauration les intéresse. Toutefois, afin de démontrer le potentiel du modèle économicoécologique, le choix de l'allocation a été décidé arbitrairement dans cette étude au prorata du tonnage de marchandises transportées annuellement par voie d'eau par chaque secteur.

Dans les Figures 8 à 10, les résultats des deux scénarios de restauration sont comparés au scénario BAU à l'horizon 2015. Toutes les valeurs sont exprimées en base $100=$ BAU, c'est-à-dire que les valeurs du scénario BAU sont fixées à 100. Par exemple, avec une valeur de PRB (Produit Régional Brut) de $30.000 \mathrm{M}_{2007}$ dans un des scénarios de restauration et de $40.000 \mathrm{M}_{2007}$ dans le scénario BAU, la valeur du scénario de restauration standardisée en base 100 deviendrait $75 \%=100 / 40000 \mathrm{M€} * 30000$.

Le modèle permet de calculer trois types d'impacts de la restauration de nourriceries : un impact écologique calculé en unité physiques et un impact macro-économique ainsi que sectoriel tous les deux calculés en unités monétaires.

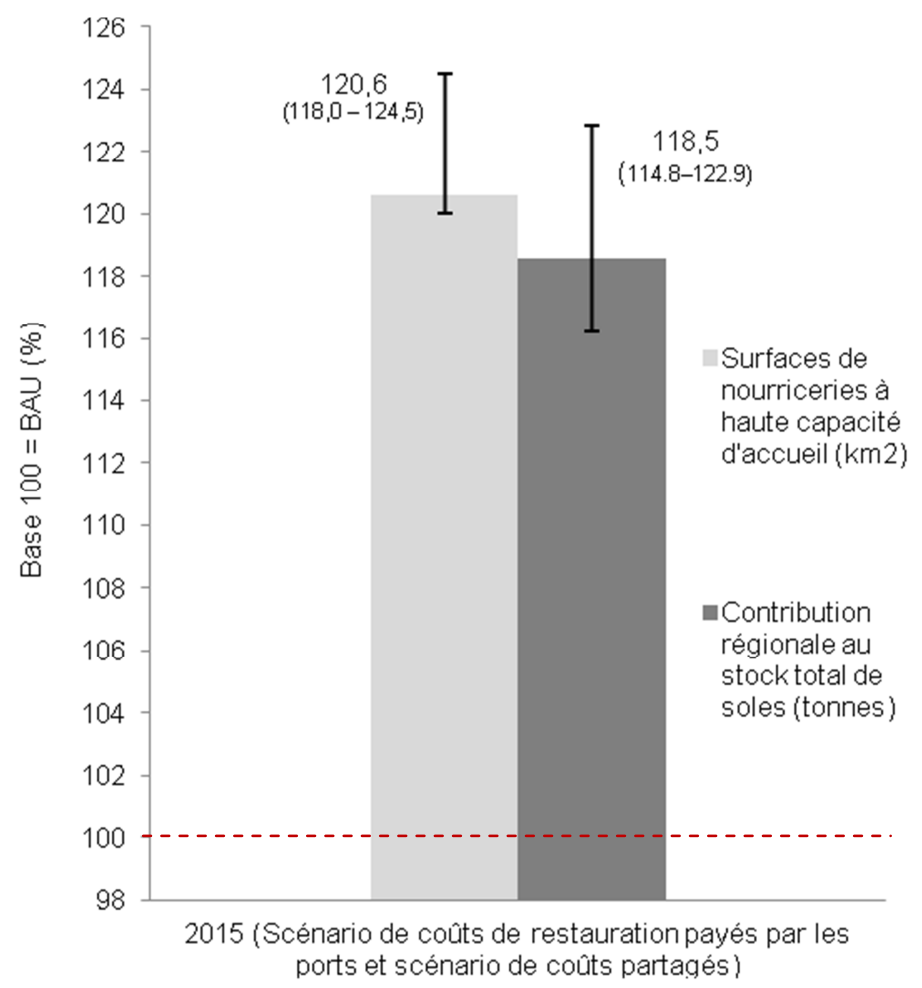

Figure 8: Impact environnemental de la restauration de nourriceries potentielles sur la période 2004-2015. Les marges d'incertitude sont indiquées entre parenthèses.

Concernant l'impact écologique, la Figure 8 montre que les deux scénarios de restauration contribuent à augmenter la surface de nourriceries en 2015 de 20,6\% par rapport à une situation en 2015 sans programme de restauration (scénario BAU). Cela correspond à une surface de $118,24 \mathrm{~km}^{2}$ pour le scénario BAU et de $142,62 \mathrm{~km}^{2}$ pour les deux scénarios de restauration. Une analyse de sensibilité sur les données associée au pourcentage d'erreur sur l'architecture du modèle montre que la hausse de la surface de nourriceries varie en réalité au sein de marges d'incertitude se situant entre une augmentation de 18,0\% à 24,5\% par rapport au BAU (barre d'erreur de la Figure 8). En insérant ces valeurs de surface dans les équations écologiques exogènes à la matrice input-output, est obtenue la biomasse de soles fournie par la Haute-Normandie à la Manche-Est.Les deux scénarios de restauration de nourriceries potentielles dépassent le scénario BAU de 18,5\% (14,8\%-22,9\%). 
L'impact macro-économique peut être signalé, comme dans la Figure 9, par trois indicateurs : le PRB, l'excédent brut d'exploitation (bénéfice des entreprises) et le salaire brut total perçu par l'ensemble des ménages.

Les deux scénarios de restauration fournissent des résultats quasiment identiques par rapport au BAU. En résultent une diminution de $-0,3 \%$ pour le PRB $(-3,1 \%-0,4 \%)$ et une perte de $-0,4 \%$ pour l'excédent brut d'exploitation $(-5,8 \%--0,4 \%)$. Concernant le revenu total des ménages perçu sous forme de salaires bruts, les deux scénarios de restauration génèrent une perte de $0,5 \%(-5,8 \%--0,4 \%)$ par rapport au scénario BAU. Cela signifie que sans mesure d'accompagnement, la restauration de nourriceries générera en 2015 0,5\% de postes de travail en moins que le scénario BAU. Ces valeurs ont été estimées sur base de l'hypothèse posée, à savoir que les entreprises porteront la charge des coûts de restauration pour moitié sur les bénéfices et pour moitié sur les coûts salariaux.

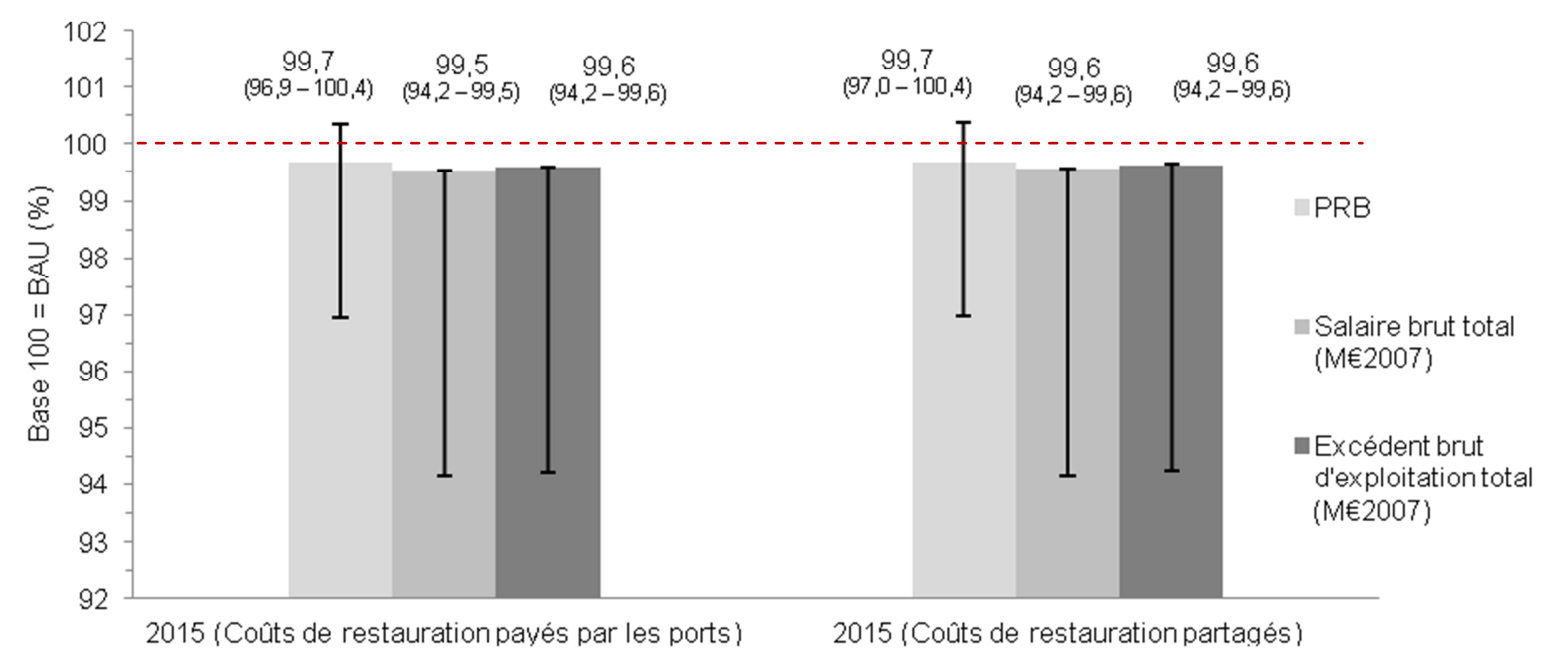

Figure 9: Impacts macroéconomiques des scénarios de restauration des nourriceries potentielles (2004-2015). Les marges d'incertitude sont indiquées entre parenthèses.

CORDIER (2011) examine également l'impact sectoriel des scénarios contrastés.Le coût direct des scénarios de restauration est assez conséquent. Il s'élève à $386 \mathrm{M}_{2007} / \mathrm{an}$, soit $0,8 \%$ du PRB de Haute-Normandie en 2007. Les impacts indirects sont, quant à eux, mitigés puisque, alors que certains secteurs voient leurs bénéfices diminuer, le secteur de la pêche et de la construction les voient augmenter. En effet, le secteur de la construction est le grand bénéficiaire des programmes de restauration.

En revanche, des impacts négatifs se font également sentir dans certains secteurs. La restauration de nourriceries potentielles génère des coûts élevés pour trois secteurs : les ports, les mines (y sont inclus l'extraction de produits miniers énergétiques comme le pétrole et le gaz naturel mais aussi des produits non énergétiques comme le sable) et le secteur des manufactures de coke, raffineries de pétrole et de combustibles nucléaires. Les ports perdent $43 \%$ de leur excédent brut d'exploitation quand ils assument seuls les coûts de restauration (Figure 10). 


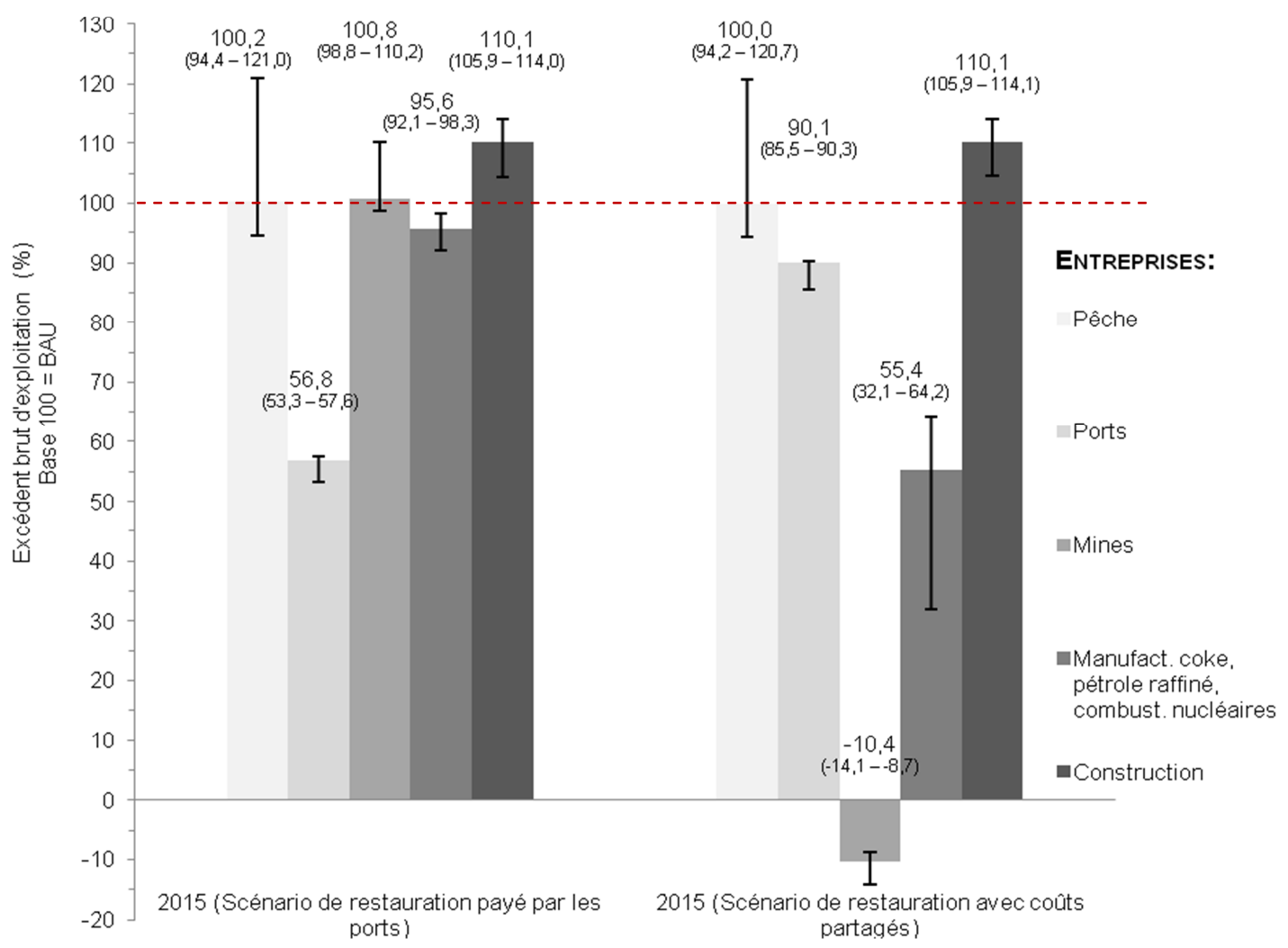

Figure10 : Impact sectoriel des scénarios de restauration de nourriceries potentielles (2004-2015) en termes d'excédent brut d'exploitation (bénéfices des entreprises). Les marges d'incertitude sont indiquées entre parenthèses.

\subsection{Une analyse "intégrée" volontairement partielle}

Le modèle «Eco » est structuré de manière à expliciter clairement les compartiments de cette réalité tridimensionnelle qui est couverte selon la méthodologie exposée à la Section 2. Il propose une désagrégation de la problématique des pressions environnementales et de la durabilité des services environnementaux, en mettant l'accent sur quelques éléments clés :

- Le compartiment environnemental de la lithosphère sous-marine (Tableau 2), et plus spécifiquement une partie de ce compartiment que sont les habitats marins de type nourriceries de poissons.

- Le compartiment environnemental de la biosphère, et plus spécifiquement la population de soles communes (Solea soleasp.).

- L'estimation des "terres importées" dans l'empreinte écologique de la métropole parisienne en provenance du sous-bassin Seine aval (et plus spécifiquement de la région Haute-Normandie).

- L'ensemble des secteurs économiques ; ceux-ci se retrouvent intégralement représentés dans la structure de base de la table input-output qui est au cœur du modèle économico-écologique régional «Eco ».

- Quelques exemplaires des "services écosystémiques" catégorisés au Tableau 1, notamment : le service de support fonctionnel et de vie offert par les habitats marins pour les soles; le service d'appropriation matérielle, le flux 
Economie $\rightarrow$ Environnement que représente l'extension des infrastructures portuaires sur les habitats marins; et le service de flux Environnement $\rightarrow$ Economie qui est la pêche annuelle de poissons marins.

Le modèle «Eco » propose ainsi une désagrégation spécifique et partielle au niveau du sous-bassin Seine Aval.Car celui-ci couvre des services écosystémiques intéressants fournis à la métropole parisienne qui ne sont pas (ou très peu) présents ailleurs.

Premièrement, le sous bassin Sein aval et son estuaire servent de «tuyau d'évacuation » des égouts de la métropole parisienne et même de l'ensemble du Grand Paris qui se déversent tous dans la Seine et finissent par être déversés dans la manche au niveau du Port du Havre. Cela affecte lourdement la capacité d'accueil en poissons des habitats marins. Celle-ci à fortement baissée depuis la révolution industrielle (CORDIER et al., 2011 ; ROCHETTE et al., 2010).

Deuxièmement, l'estuaire de la Seine offre à la métropole parisienne un service de support (la voie fluviale) pour les produits transportés (énergies fossiles, produits miniers, agricoles, halieutiques, etc.) via le port du Havre et de Rouen par exemple. Il existe une réelle dépendance de la métropole parisienne par rapport à la mer en ce qui concerne ses approvisionnements en produits français. L'estuaire de la Seine approvisionne la métropole parisienne à raison de $11 \%$ de ses importations interrégionales ${ }^{12}$ en énergies transformées (pétrole raffiné, charbon cokéfié, uranium transformé) et à raison de $22 \%$ de ses importations interrégionales en poissons (calculs propres sur base des données du MEEDDAT, 2009). Le revers de la médaille, c'est la destruction des habitats marins de l'estuaire de la Seine par l'extension des infrastructures portuaires.

12 Les importations interrégionales sont internes à la France et proviennent d'une des 22 régions métropolitaines (Corse incluse). 


\section{- 5 - \\ Deux outils complémentaires, pour mieux articuler les échelles globale et locale d'évaluation}

Nous discutions dans cette Section la portée (et les limites) des deux modalités d'analyse en termes de leur complémentarité.

\subsection{Analyse désagrégée et vision holiste}

L'estimation d'une empreinte environnementale, comme exposée au Tableau 4 permet de détailler l'information contenue implicitement dans l'empreinte écologique et de montrer la responsabilité de chaque produit économique dans cette empreinte. Un des produits à la responsabilité la plus élevée sont les services.En effet, en sommant l'empreinte environnementale des différents services (depuis la construction jusqu'aux services pour les ménages), on arrive à 48,3\% de l'empreinte total de la métropole parisienne. Cela s'explique par deux éléments.

- Premièrement, l'efficience écologique des services est relativement faible par rapport aux secteurs secondaires (voir les secteurs industries et automobile au Tableau 5). Par conséquent, il faut une quantité importante de surfaces pour produire une unité monétaire (1 Euro) de service par le secteur tertiaire.

- Deuxièmement, la valeur monétaire totale produite par le secteur tertiaire est très élevée car ce secteur est prédominant dans l'économie française (80\% du PIB). Cela augmente d'autant plus la quantité de surfaces nécessaires pour le fonctionnement de l'économie.

Cette information est utile pour dresser un programme de mesures environnementales efficace qui cible spécifiquement le secteur des services.

Cependant, si l'empreinte environnementale est capable de révéler l'importance du secteur des services dans la pression environnementale causée par la métropole parisienne sur les régions extérieures, elle ne peut toutefois pas mettre en évidence le rôle du service portuaire dans la consommation de surfaces de terres ni spécifier que ces surfaces concernent des habitats marins de l'estuaire de la Seine (localisé dans le sous-bassin Seine aval - $c f$. Figure 1). D'où l'intérêt du modèle «Eco» qui, de par sa désagrégation sectorielle et spatiale, peut non seulement identifier le rôle des ports mais également le quantifier.

Plus particulièrement, le modèle «Eco» permet de quantifier le rôle des secteurs économiques indirectement responsables de la dégradation des habitats marins par l'usage des services portuaires. La modélisation révèle, par exemple, que le secteur minier (minerais sableux, pierreux et métalliques) et celui de l'extraction et du traitement des ressources énergétique du sous-sol (extraction de charbon, gaz, pétrole et uranium, raffineries de pétrole et traitement du charbon et de l'uranium) sont des responsables indirects très important de l'empreinte écologique.

L'empreinte environnementale le révèle également, en partie, comme signalé au Tableau 4 : les «autres produits primaires » qui regroupent ces deux secteurs représentent $9,7 \%$ de l'empreinte, ce qui en fait les $4{ }^{\text {ème }}$ plus gros contributeurs à l'empreinte sur un total de 17 catégories de produits économiques. Ceci dit, l'empreinte environnementale ne permet 
pas de savoir s'il s'agit de produits agricoles ou horticoles, de minerais sableux, pierreux ou métalliques, d'énergies fossiles ou d'uranium. Le modèle «Eco » offre un découpage plus désagrégé sectoriellement. Il nous apprend que les industries qui utilisent majoritairement les services portuaires sont au nombre de deux : l'industrie des produits miniers (sables, minerais métalliques, pierres) et l'industrie de l'extraction et du traitement des ressources énergétiques du sous-sol, des énergies fossiles brutes (charbon, gaz naturel, etc.) et du pétrole raffiné. Ces deux secteurs industriels représentent ensemble $78 \%$ du total des marchandises transportées par voie d'eau dans l'estuaire de la Seine en 2006 (CORDIER, 2011, p. 350). Ces produits sont donc le moteur de l'activité économique portuaire dont l'empreinte sur les habitats marins est conséquente. Dans un sens, le calcul de l'efficience écologique (output (€)/empreinte (hag)) l'indiquait déjà au Tableau 5 : l'efficience du secteur minier (extraction de sables, pierres, et minerais métalliques)est très faible : $253354 € / \mathrm{hag}$. Cela signifie que le secteur minier doit consommer énormément de surfaces de terrains pour produire une unité de minerais (une tonne ou un euro).

L'indicateur d'efficience écologique que nous venons d'illustrer, ne permet pas de mettre en évidence la responsabilité du secteur de l'extraction et du traitement des ressources énergétiques du sous-sol dans la consommation de terres d'habitats marins. Son efficience écologique (Tableau 5) s'élève à 2614650 €/hag. C'est une valeur élevée qui suggère que le secteur est peu consommateur de surface de terrains et que, par conséquent, son empreinte écologique est susceptible d'être faible. Cela est vrai si l'on ne considère que la responsabilité directe de ce secteur. Par contre, en considérant sa responsabilité indirecte dans la destruction des nourriceries de par le fait qu'il consomme du service portuaire pour transporter ses marchandises par voie d'eau, le secteur de l'extraction et du traitement des ressources énergétiques du sous-sol est un responsable important de la consommation de terres d'habitats marins. Cette responsabilité indirecte peut-être évaluée par le pourcentage de marchandises qu'il transporte par voie d'eau dans le total des marchandises transportées par voie d'eau dans l'estuaire de la Seine en 2006. Ce pourcentage s'élève à 66\% (CORDIER, 2011, p. 350).

\subsection{Indicateurs et Aide à la Décision}

In fine, l'un des points forts du modèle «Eco » est de permettre d'identifier les secteurs économiques responsables directement et indirectement de "terres importées" dans l'empreinte écologique par la métropole parisienne depuis la périphérie ouest du Grand Paris. Cela permet ensuite de réfléchir à un programme de mesures précis qui soit à la fois écologiquement efficace et socialement juste. Des mesures efficaces peuvent être identifiées par le modèle «Eco » précisément parce qu'il peut quantifier la relation entre les activités économiques et les surface d'habitats marins. Cela permet de cibler les activités économiques à viser préférentiellement pour agir significativement sur les terres sous-marines d'habitats marins. Quant aux mesures justes, elles peuvent être identifiées par le modèle dans le sens où il est capable de quantifier les responsabilités directes et indirectes de la dégradation des habitats. Cela permet par la suite de déclarer un principe d'équité et, calculer une clé de répartition du coût de la mesure entre secteurs au prorata de leur contribution directe et indirecte dans la consommation de terres d'habitats marins.

En effet, le modèle «Eco » constitue un outil d'aide à la délibération, dans la mesure où il permet d'identifier les compromis envisageables, de repérer les secteurs les plus vulnérables dans ce compromis et de quantifier l'ampleur de celui-ci en termes monétaires pour les secteurs économiques (excédent brut d'exploitation, valeur ajoutée, emploi) et en unités 
physiques pour l'environnement (tonnes de soles, hectares de nourriceries). Ces atouts offrent des avantages potentiels pour des processus de décision participatifs.

Les impacts sectoriels négatifs estimés par le modèle «Eco » illustrent tout l'enjeu politique de la «distribution de sacrifice» pour que la restauration de l'écosystème soit réalisée. Par accord négocié ou de fait, un «compromis» doit être opéré entre — parmi d'autres - l'objectif de maximisation des profits des entreprises et l'objectif environnemental de restauration de nourriceries potentielles. A ce propos, des pratiques de délibération publique peuvent viser à prendre en compte la multiplicité des perspectives légitimes afin de trouver un "compromis".

Il est inévitable que des polémiques naissent et opposent les perspectives des acteurs vivant dans des réalités sociales et des missions économiques distinctes (O'CONNOR, 2000 ; O’CONNOR, 1999 ; BREMER, 2010). Sans prétendre de résoudre définitivementles conflits, les approches de décision participative peuvent permettre de rendre les compromis plus transparents pour les décideurs en identifiant les acteurs et les ressources avantagés et désavantagés. Munda et al. (1994) et VAN DEN BERGH et NIJKAMP (1991) estiment que rendre les compromis clairs et apparents aux acteurs est une condition quasi nécessaire pour que les mesures de gestion environnementale soient opérationnelles. Rendre les compromis plus transparents trouve aussi son sens dans l'application de valeurs démocratiques (STIRLING, 2006; O'NEILL, 2007). Cela part du principe qu'une démocratie n'est pleinement aboutie que quand les citoyens participent à l'organisation de la cité et ne sont pas réduits au seul rôle d'électeurs (DRYZEK, 2002 ; BREMER, 2010). Enfin, rendre les compromis clairs et apparents permet d'identifier les acteurs défavorisés par les mesures ou projets politiques en vue d'éventuellement les compenserpour éviter que leur qualité de vie ou leur activité économique ne périclite (exonération fiscale, subsides compensatoires, etc.).

Il existe de nombreuses approches de délibération participatives. Celle expérimentée par CORDIER (2011), dénommée INTEGRAAL, propose d'impliquer les acteurs dans un processus d'évaluation multicritère, multi-scénario et multi-acteur décision comportant 5 étapes $^{13}$. Ce qui est présenté ci-dessous constitue un exemple hypothétique d'application destiné à illustrer le champ des politiques possibles autour de l'estuaire de la Seine.

La première étape d'INTEGRAAL vise à préciser le problème de conflit ou de décision à résoudre.Une illustration de conflit potentiel est donnée dans ce qui suit. Les pêcheurs professionnels et les pêcheurs de loisir pourraient s'associer avec la Maison de l'Estuaire (association de préservation de l'environnement) pour s'opposer aux activités portuaires.Cette coalition serait par exemple en mesure d'argumenter que les ports devraient restaurer une plus grande surface de nourriceries afin d'accroître la population des jeunes alevins produits dans l'estuaire de la Seine. Le secteur portuaire pourrait rétorquer aux pêcheurs que la pêche professionnelle constitue la principale cause de diminution des stocks de poissons marins en Europe. En conséquence, avant de clamer la nécessité de restaurer des surfaces de nourriceries, il serait bon de réduire l'effort de pêche....

La deuxième étape de la démarche INTEGRAAL consiste à imposer la structure d'un problème de choix social: en termes d'acteurs (les parties prenantes de la problématique considérée), de catégories d'enjeux de performance (ex.: la pérennité des services écosystémiques, la viabilité et la rentabilité économique, l'acceptabilité sociale, etc.) et d'options d'actions à mettre en œuvre (options technologiques, régimes de gouvernance, mécanismes d'incitation à évaluer).

$13 \quad$ Il existe en outre une vaste littérature sur l'analyse intégrée et les démarches délibératives, citons par exemple :CHAMARET et al. (2007) ; O'CONNOR \& SPANGENBERG (2007); Holland (1997) ; FAUCHEUX \& HuE (2001) ; FRAME \& BROWN (2008); O’NEILL (2007). 
Encadré 1. Structuration de la problématique en 3 scénarios, 5 acteurs et 5 enjeux de performance (ou critères d'évaluation).

- Les options envisagées sont illustrés par les 3 scénarios exposés plus haut, à savoir : le scénario sans mesures de restauration (BAU) et les deux scénarios de restauration de nourriceries.

- Les acteurs principaux impliqués dans la problématique des nourriceries de l'estuaire de la Seine peuvent être identifiés comme : (i) les raffineries de pétrole et producteurs de gaz et de charbon, (ii) les ports du Havre et de Rouen, (iii) les pêcheurs professionnels et de loisir, (iv) la Maison de l'Estuaire (association sans but lucratif chargée de la gestion de la réserve naturelle de l'estuaire de la Seine), (v) le secteur de la construction (pour mettre en œuvre la restauration de surfaces de nourriceries).

- Les enjeux de performance pour la comparaison des scénariosdevraient couvrir toutes les dimensions du développement territorial.Un premier enjeu est articulé en termes de l'état de l'environnement: en surface de nourriceries ainsi qu'en biomasse totale de la population de soles produite par l'estuaire de la Seine.Un deuxième enjeu de performance est celui de la rentabilité économique des secteurs. Cette rentabilité peut êtresignalée par l'excédent brut d'exploitation (les bénéfices).Ensuite, un enjeu d'acceptabilité sociale pourrait être appréhendé par le nombre d'emplois.Et enfin, la performance macroéconomique régionale serait, quant à elle, appréhendée par le Produit Régional Brut, un indicateur du niveau de la production économique et des revenus produits en Haute-Normandie.

La problématique des nourriceries peut donc être structurée en 5 catégories d'acteurs, 3 scénarios de mesures de gouvernance et5 critères d'évaluation que sont les enjeux de performance (Encadré 1).

La troisième étape de la démarche INTEGRAAL a pour objectif de mobiliser des outils pour la représentation du système et l'estimation d'indicateurs pour chaque scénario. Dans le cas présent, l'outil principal utilisé est le modèle «Eco » (CORDIER, 2011).

La quatrième étape consiste à mobiliser les acteurs pour une évaluation multicritère des options d'actions.Pour le présent exemple, cette structuration est résumée par trois tableaux (un par scénario) de 25 cellules chacun, avec, en colonne, les 5 critères d'évaluation et en ligne les 5 catégories d'acteurs ( $c f$. Tableau 6, qui constitue l'une des 3 tranches de la Matrice de Délibération $)^{14}$. Les 5 catégories d'acteurs sont donc amenées àconsidérer les 5 enjeux de performances pour comparer et évaluer les 3 options d'actions. Le Tableau 6 illustre un cas hypothétique dans lequel nous avons imaginés quelles pourraient être les réactions des acteurs au moment d'évaluer l'option «mesure de restauration avec partages des coûts » (dans ce scénario, les coûts de restauration des nourriceries sont répartis équitablement entre responsables directs et indirects).

La cinquième et dernière étape d'INTEGRAAL consiste à communiquer les résultats de l'étude auprès des acteurs ayant participé à la délibération mais aussi auprès du public de façon plus large.In fine, il s'agit de passer de la recherche au processus de délibération, de débat, de décision. La démarche présentée ci-dessus permet d'identifier deux lignes potentielles de conflit d'usage de l'estuaire.

Une première ligne de conflit opposerait les associations de défense de l'environnement (ex. Tableau 6: Maison de l'estuaire) aux responsables directs et indirects de la destruction des nourriceries (Ports du Havre et de Rouen et industries bénéficiant des services portuaires pour le transport de marchandises - ex. Tableau 6: Raffineries de pétrole et producteurs de gaz et de charbon).

La deuxième ligne de conflit opposerait les Pêcheurs professionnels et de loisir aux responsables directs et indirects.

14 Pour les principes généraux de fonctionnement de la matrice de délibération et, son encastrement dans la démarche INTEGRAAL, voir DA CUNHA (2010). Pour les principes et l'utilisation de la version en ligne, voir BUREAU et al. (2007), MAXIM \& O'CONNOR (2009); et le site kerDST at http://kerdst.kerbabel.net. 
Ces deux lignes de fracture reflètent un conflit d'usage de l'estuaire du Grand Paris entre, d'une part, les acteurs qui utilisent ou défendent les services écosystémiques de support de vie (habitats de nourricerie de poissons et d'oiseaux limicoles) et ; d'autre part, ceux qui ont recours à l'estuaire pour ses services écosystémiques d'approvisionnement en eau utilisée comme moyen de transport (importations et exportations par bateau).

Tableau 6. Scénario avec mesures de restauration des nourriceries et coûts partagés : évaluation multicritère et multiacteur hypothétique en Haute-Normandie

\begin{tabular}{|c|c|c|c|c|c|c|}
\hline & \multicolumn{6}{|c|}{ CRITÈRES D'ÉVALUATION (ENJEUX DE PERFORMANCE) } \\
\hline & & ETAT DE & NVIRONNEMENT & $\begin{array}{l}\text { RENTABILITÉ } \\
\text { FINANCIĖRE }\end{array}$ & $\begin{array}{l}\text { ACCEPTABILITÉ } \\
\text { SOCIALE }\end{array}$ & $\begin{array}{c}\text { Production } \\
\text { ÉCONOMIQUE GLOBALE }\end{array}$ \\
\hline \multirow{6}{*}{ 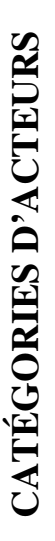 } & & $\begin{array}{l}\text { Surface de } \\
\text { nourriceries } \\
\left(\mathrm{km}^{2}\right)\end{array}$ & $\begin{array}{l}\text { Population de soles } \\
\text { de l'estuaire } \\
\text { (tonnes) }\end{array}$ & $\begin{array}{l}\text { Bénéfices } \\
\text { sectoriels } \\
\text { (M€ } €_{2007)}\end{array}$ & $\begin{array}{l}\text { Nombre total } \\
\text { d'emplois }\end{array}$ & $\begin{array}{l}\text { Produit Régional Brut } \\
\text { (M€ 2007) }\end{array}$ \\
\hline & $\begin{array}{l}\text { Raffineries de pétrole et } \\
\text { producteurs de gaz et de } \\
\text { charbon }\end{array}$ & & & & & \\
\hline & $\begin{array}{l}\text { Ports du Havre et de } \\
\text { Rouen }\end{array}$ & & & & & \\
\hline & $\begin{array}{l}\text { Pêcheurs professionnels } \\
\text { et de loisir }\end{array}$ & & & & & \\
\hline & Maison de l'Estuaire & & & & & \\
\hline & $\begin{array}{l}\text { Secteur de la } \\
\text { construction }\end{array}$ & & & & & \\
\hline
\end{tabular}

Clé : Cellules gris-clair $\square$ 『formance jugée acceptable par les acteurs; Cellules gris-moyen : per \\ance jugée médiocre par les acteurs mais passables; Cellules gris-foncé : $\square$ formance jugée inacceptable par les acteurs.

Une manière d'explorer un "compromis" pourrait être l'articulation d'un nouveau (troisième) scénario de restauration dans lequel, ce ne sont plus seulement les responsables directs et indirects qui assument les coûts mais également les responsables indirects de deuxième ordre. Il s'agit des secteurs des services bénéficiant des produits fabriqués par les secteurs primaires et secondaires à partir d'importations transportées par voie d'eau. La charge des coûts par secteur étant mieux répartie, elle serait fortement allégée. Par conséquent, les acteurs invoqueraient peut-être moins d'arguments financiers et économiques pour rejeter un scénario de mesures de restauration.

Le conflit qui oppose les pêcheurs aux ports, au secteur minier ainsi qu'aux raffineries de pétrole et producteurs de gaz et de charbon s'avère délicat parce qu'il est supposé que les pêcheurs font pression pour augmenter la surface de nourriceries à restaurer. Or le coût de restauration étant très important, cela pourrait mettre en péril la viabilité financière et, par conséquent, l'existence des secteurs économiques. Ceci soulève la question de la légitimité des activités de ces secteurs. Certes, d'un côté, ils sont à l'origine de la destruction de surfaces de nourriceries ainsi que de la biodiversité halieutique et avicole qu'elles abritent mais, d'un autre côté, les ports et les secteurs qui y sont liés génèrent $24 \%$ du PRB de la région Haute-Normandie ainsi que 19\% des emplois directs et indirects (CORDIER, 2011). Il est concevable que l'extension de l'allocation des coûts au secteur des services permette d'augmenter la surface de nourriceries à restaurer tout en maintenant le coût de restauration pris en charge par chaque secteur à un niveau acceptable. 


\section{- 6 - \\ Conclusion}

A l'issue de cet article, il apparaît clairement que les enjeux du développement économique et de gouvernance territoriale du Grand Paris peuvent être efficacement abordés par le biais de la dimension environnementale. L'articulation des trois outils d'analyse l'empreinte écologique de la métropole parisienne; l'empreinte environnementale de la métropole parisienne désagrégée pour 17 secteur; et, un modèle économico-écologique régional désagrégé tant sectoriellement (35 secteurs) que spatialement (estuaire de la Seine) - justifie à merveille le principe "penser globalement (le Grand Paris), agir localement (l'estuaire de la Seine) ».

L'activité portuaire, le secteur de la pêche et celui des industries de transformation de l'énergie (pétrole, charbon et uranium) de la périphérie ouest du Grand Paris sont en partie tributaires de l'activité économique de la métropole parisienne (et des autres régions aussi mais dans unemoindre mesure). C'est l'économie de la métropole parisienne qui exploite le sous-bassin Seine aval (correspond à la Haute-Normandie) en achetant les produits acheminés et transformés dans ses ports. Cela génère une délocalisation des pressions environnementales de la métropole parisienne vers la périphérie maritime du Grand-Paris. C'est ce qu'on appelle dans le langage de l'empreinte écologique des "terres importées" par la métropole parisienne.

L'empreinte environnementale apporte une précision à la fenêtre d'analyse fournie par l'empreinte écologique. Son niveau de désagrégation en 17 types de produits économiques domestiques et importés permet à la fois d'estimer la contribution de chaque produit à l'empreinte environnementale et de distinguer la contribution due à des produits fabriqués sur le territoire de la métropole parisienne $(59 \%)$ de la contribution due à des produits importés $(41 \%)$ - les pressions environnementales engendrées par ces produits sont appelées «terres importées ». L'empreinte environnementale désagrégée par produits permet donc d'évaluer l'extension de l'emprise de l'économie de la métropole parisienne sur les surfaces de terres des autres régions du Grand Paris en estimant la valeur des terres importées. Elle permet également d'identifier les produits qui contribuent le plus à l'empreinte (services, produits miniers et ressources énergétique du sous-sol) sur lesquelles les mesures environnementales devraient être ciblées pour réduire efficacement les pressions environnementales des activités économiques.

Cependant, un niveau supplémentaire de désagrégation est nécessaire pour informer les processus de décision à l'échelle d'un secteur ou d'un territoire spécifique. Nous avons illustré cette nécessaire désagrégation par la problématique des «boucles » d'influence entre le Grand Paris et les écosystèmes productifs de son estuaire. La modélisation «Eco » est à la fois intégrée et partielle. Elle cible une seule catégorie de terres : les habitats marins de l'estuaire de la Seine. Ces terres doivent représenter une proportion minime de l'empreinte environnementale de la métropole parisienne (certainement beaucoup moins de 5\%, à en juger par la contribution des terres de pêches au Tableau 3). Cependant la destruction des habitats marins de la région Haute-Normandie posent de réels soucis à l'échelle régionale: l'estuaire de la Seine constitue un vivier très important de poissons pour la zone de pêche internationale située dans la Manche (CoRDIER et al., 2011). Dans ce contexte, le modèle «Eco » permet 
d'identifier les "responsables indirects" et ensuite de réfléchir à une clef d'allocation du coût des mesures à adopter.

Peut-on réellement espérer un "Grand Paris de demain», respectueux de l'environnement? Peut-on envisager un «développement durable» des ressources halieutiques côtières ? Comment éviter que les «services environnementaux » de l'estuaire en aval de Paris, démontrés scientifiquement comme importants directement et indirectement pour des activités de pêche (et donc, pour contribuer à nourrir la population francilienne), ne sombrent dans un dilemme de prisonnier? Pour répondre aux objectifs ambitieux d'aménagement de Paris Métropole et encore davantage du Grand Paris, il devient indispensable de constituer des connaissances sur les interdépendances et les marges de manœuvre. Les méthodes et modèles tels que ceux exposés dans cet article, ne peuvent pas aboutir à des représentations complètes et exactes. Néanmoins, ils permettent d'explorer des options politiques et peut-être de nourrir un climat de négociation nécessaire à la résolution des conflits d'usages.

\section{Bibliographie}

ANDRES L, BROCHET B (2010) Ville durable, ville mutable : quelle convergence en France et en Suisse ? Revue d'Economie Régionale Urbaine 4: octobre 2010.

AUDRAIN J, GUYONNAUD M-F (2011) L'empreinte environnementale appliquée sur des territoires. @ Editions T.I.

Audrain, J, Cordier M, Faucheux S, GuYonnaud M-F, Nicolaï I, O'Connor M (2011), La Ville et son Estuaire: Ecologie territoriale et Indicateurs pour un Développement Durable de la Métropole Parisienne, REEDS Working Papers / Cahiers de REEDS No.2011-11 (septembre 2011), UVSQ, Rambouillet.

BicKNell KB, BALl RJ, CULLEN R, BigsBy HR (1998) New methodology for the ecological footprint with an application to the New Zealand economy. Ecological Economics 27: 149-160.

BOUTEAU A, GONDRAN N(2009) L'empreinte écologique. Collection Repères n 527 : février.

BREMER S (2010) Mobilising high-quality knowledge through dialogic environmental governance: a comparison of approaches \& institutional settings. A paraître IJSD (2013). Disponible comme: Cahiers de REEDS No. 2010-09, UVSQ, Rambouillet, 26 pp.

BuREAu P, ReICHEL V, LEgRAND F, O'CONNOR M, DA CUNHA C (2007) Manuel d'utilisateurs pour KerDST, le système d'aide à la délibération en-ligne. Cahiers du C3ED, C3ED - Université de Versailles Saint-Quentin-EnYvelines, Guyancourt, France.

CARPENTIER CL(1994) Agriculture and the environment: an economic-ecological Input-Output Model of the Canadian economy. PhD at McGill University Montreal,121 pp.

CASTELLS M (2001) La société en réseaux. L'ère de l'information, Nouvelle édition, Paris, Fayard.

ChAmARET A, O'ConNor M, ReCOCHE G, (2007). Top-down/bottom-up approach for developing sustainable development indicators for mining: application to the Arlit uranium mines (Niger). International Journal of Sustainable Development 10: 161-174.

CORDIER M (2012). Intégration d'un service écosystémique intermédiaire à une modélisation input-output économico-écologique : cas de de l'estuaire de la Seine. In : HAMAIDE B, BRUnET S (Ed.), Développement durable et économie régionale. Publication des FUSL, Bruxelles, Belgique.

CORDIER M (2011) Ecosystème estuarien et système économique régional: faisabilité d'une intégration par modélisation Input-Output. Application au cas de l'habitat halieutique dans l'estuaire de la Seine. Thèse de doctorat à l'Université Libre de Bruxelles et à l'Université de Versailles-Saint-Quentin-en-Yvelines, 477 pp.

CORDIER M, PÉREZ AGÙNDEZ JA, O'CONNOR M, ROCHETTE S, HECQ W (2011). Quantification of interdependencies between economic systems and ecosystem services: an input-output model applied to the Seine estuary. Ecological Economics70(9):1660-1671. 
Cuvilliez A, Deloffre J, LAFITE R, Bessineton C (2009) Morphological responses of an estuarine intertidal mudflat to constructions since 1978 to 2005: The Seine estuary (France). Geomorphology 104 (3-4): 165-174.

DA CUNHA C (2010). Quelle prise en compte de la biodiversité dans les enjeux de performances des activités agricoles en Ile-de-France? Expérimentation d'une méthode d'évaluation participative multicritères. Thèse de doctorat en Sciences économiques. Université de Versailles Saint-Quentin-en-Yvelines, Guyancourt, France, 395 pp.

DALY HE(1968) On Economics as a Life Science. Journal of Political Economics 76: 392-406.

DE GRoOT RS, WiLSON MA, BoumANS RMJ (2002) A typology for the classification, description and valuation of ecosystem functions, goods and services. Ecological Economics 41: 393-408.

DECAMPS M, ViCARD F (2010) Mesurer le développement durable : jeux d'indicateurs et enjeux locaux. Revue d'Economie Régionale Urbaine 4: octobre.

DRYZEK, J.S., 2002. Deliberative democracy and beyond: liberals critics and contestations. New York, Cambridge University Press.

Ducrotoy J-P, DAUVIN J-C (2008) Estuarine conservation and restoration: The Somme and the Seine case studies (English Channel, France). Marine Pollution Bulletin 57:208-218.

EWING B, REED A, RizK SM, GALli A, WACKERNAGEL M, KITZES J (2008) Calculation methodology for the 2008 national footprint accounts. Oakland (CA), Global Footprint Network.

FAucheuX S, HuE C (2001) From Irreversibility to Participation: Towards a participatory foresight for the governance of collective environmental risks. Journal of Hazardous Materials 86: 223-243.

Faucheux S, O’CONNOR M (eds.) (1998) Valuation for Sustainable Development: Methods and Policy Indicators, Edward Elgar, Cheltenham.

FISCHER B, TURNER KR, MORLING P (2009) Defining and classifying ecosystem services for decision making. Ecological Economics 68: 643-653.

FONDATERRA, 2010. Bilan carbone ${ }^{\circledR}$ territorial, empreinte environnementale et test des indicateurs Agenda 21 du MEEDM de la communauté d'agglomération du plateau de Saclay. CAPS.

FRAME B, BROWN J (2008) Developing post-normal sustainability technologies. Ecological Economics 65: 225241.

GiAMPIETRO M, MAYUMI K, MUNDA G (2006) Integrated assessment and energy analysis: quality assurance in multi-criteria analysis of sustainability. Energy 31: 59-86.

Grumbach A (2008)Paris, Rouen, Le Havre, une seule ville dont la Seine est la grande rue. Ministère de la Culture et de la Communication. EMOC Etablissement Public de Maîtrise d'Ouvrage des Travaux Culturels.

HAAKE J (2000) Les stratégies des entreprises pour une utilisation des matières plus respectueuse de l'environnement : une application du concept de dématérialisation à la gestion environnementale des entreprises industrielles. Thèse doctorat à l'Université de Versailles-St-Quentin-en-Yvelines.

HAILS C, LOH J, GOLDFINGER S (2006) Living planet report 2006. World Wide Fund for Nature International (WWF), Zoological Society of London (ZSL), Global Footprint Network.

Holland A (1997) The foundations of environmental decisionmaking. International Journal of Environment and Pollution 7: 483-496.

IAU (2011) Les indicateurs synthétiques de l'environnement en Ile de France. Mémento, étude n799.

ICES, accédé en 2009. Stock Assessment Summary/Standard Graph Database. URL: http://www.ices.dk/datacentre/StdGraphDB.asp

ICES, 2008.Report of the ICES Advisory Committee 2008.ICES Advice, 2008.Book 6.Denmark, 326 pp.

IFEN (2001) Développement durable et capital naturel critique, concepts et consultation d'experts. Collection Etudes et Travaux, ${ }^{\circ} 32$, Orléans.

ISARD W (1968) Some Notes on the Linkage of Ecological and Economic Systems. Regional Science Association, Papers, XXII, Budapest conference.

Jin D, Hoagland P, DaLton TM (2003) Linking economic and ecological models for a marine ecosystem. Ecological Economics 46: 367-385.

LEROND M (2006). Profil environnemental régional de Haute-Normandie. Préfecture de la Région HauteNormandie.

MAXIM L, O'CONNOR M (2009) The use of the Deliberation Matrix for building a system of survey and the foresight of changes in biodiversity in the Île-de-France region (France). In: RoDRIGUEZ-LABAJOS B, Spangenberg JH, Maxim L, Monterroso i, Binimelis R, Martinez Alier J, Kuldna P, Peterson K, 
Uustal M, Gallai N (eds.), Assessing biodiversity risks with socio-economic methods: ALARM experience, Pensoft Publishers, Sofia.

MCDONALD G (2005) Integrating economics and ecology : a systems approach to sustainability in the Auckland region. $\mathrm{PhD}$ thesis at Massey University, Palmerston North, New Zealand.

MEEDDAT (2009).Transport national et Transport international en 2006.Banque de donnéesSitraM. Données transmises par courrier postal le 24 juin. Demande $n^{\circ} 09031 /$ devis $n^{\circ} 2564$.

MCDONALD GW,PATTERSON MG (2004) Ecological Footprints and Interdependencies of New Zealand Regions. Ecological Economics 50: 49-67.

Millennium Ecosystem Assessment (2005) Ecosystems and Human Well-being: Synthesis. Island Press (Ed.), Washington DC.

MiLLER RE, BLAIR PD (2009) Input-output Analysis. Foundations and Extensions. Cambridge University press, United-Kingdom.

Ministere DE L'Egalite Des TerRitoires ET DU Logement(accédé en 2012) Le Grand Paris. URL http://www.mon-grandparis.fr/le-grand-paris/description-generale

MUNDA G., NIJKAMP P, RIETVELD P (1994) Qualitative multicriteria evaluation for environmental management. Ecological Economics 10: 97-112.

MUNDA G. (2004). Social multi-criteria evaluation: methodological foundations and operational consequences. European Journal of Operational Research 158, 662-677.

O'Connor M (2000) Pathways for environmental evaluation: a walk in the (Hanging) Gardens of Babylon. Ecological Economics 34 (2), 175-193.

O'CONNOR M (1999) Dialogue and debate in a post-normal practice of science: a reflexion. Futures 31: 671-687.

O'CONNOR M, SCHOER K,(2010) Environmental degradation in the new SEEA : proposals for a systematic presentation of environmental degradation in the framework of EEA. REEDS Research Report 2010-01.

O'CONNOR M, SPANGENBERG JH(2007) A methodology for CSR reporting: assuring a representative diversity of indicators across stakeholders, scales, sites and performance issues. Journal of Cleaner Production 16(13): 1399-1415.

O’NeILL JJ (2007) Markets, Deliberation and Environment. London: Routledge.

RochetTe S, Rivot E, Morin J, MACKInSON S, Riou P, LE PAPE O (2010) Effect of nursery habitat degradation on flatfish population renewal. Application to Solea solea in the eastern Channel (Western Europe). Journal of Sea Research 64 (1-2): 34-44.

STIRLING A (2006) Analysis, participation and power: justification and closure in participatory multi-criteria analysis. Land Use Policy 23, 95-107.

VAN DEN BERGH JCJM, NIJKAMP P (1991) Operationalizing sustainable development: dynamic ecological economic models. Ecological Economics 4: 11-23.

VICTOR AP (1972a) Pollution: economy and environment. Georges Allen \&Unwin Ltd. (Eds.), Great Britain.

VICTOR AP (1972b) Economics of pollution. Macmillan studies in economics, Royaume-Uni.

WACKERNAGEL M, REES WE (1996) Our ecological footprint: reducing human impact on the earth. Philadelphia, PA: New Society Publishers.

WiEdMANN T, MinX J, BARRETT J, WACKERNAGEL M (2006) Allocating ecological footprints to final consumption categories with input-output analysis. Ecological Economics 56: 28-48.

WWF (2009) Le Grand Paris passé au crible: les propositions du WWF - France. 28 avril. URL: www.wwf.fr/s-informer/actualites/le-grand-paris-passe-au-crible-les-propositions-du-wwf-france

ZHANG Y, McDonald G, NiXON P, SMITH N (2008) Development of a regional Social Accounting Framework for New Zealand. New Zealand Centre for Ecological Economics (Eds.), Palmerston North, New Zealand. 